ARTICLE

https://doi.org/10.1038/s41467-020-19320-9

\title{
OPEN
}

\section{Natural variations of SLG1 confer high-temperature tolerance in indica rice}

\author{
Yufang Xu (1) 1,2,4, Li Zhang ${ }^{1,2,4}$, Shujun Ou ${ }^{3}$, Ruci Wang ${ }^{1}$, Yueming Wang ${ }^{1}$, Chengcai Chu (1) ${ }^{1,2 \bowtie} \&$ \\ Shanguo Yao ${ }^{1 \times}$
}

With global warming and climate change, breeding crop plants tolerant to high-temperature stress is of immense significance. tRNA 2-thiolation is a highly conserved form of tRNA modification among living organisms. Here, we report the identification of SLG1 (Slender Guy 1), which encodes the cytosolic tRNA 2-thiolation protein 2 (RCTU2) in rice. SLG1 plays a key role in the response of rice plants to high-temperature stress at both seedling and reproductive stages. Dysfunction of SLG1 results in plants with thermosensitive phenotype, while overexpression of SLG1 enhances the tolerance of plants to high temperature. SLG1 is differentiated between the two Asian cultivated rice subspecies, indica and japonica, and the variations at both promoter and coding regions lead to an increased level of thiolated tRNA and enhanced thermotolerance of indica rice varieties. Our results demonstrate that the allelic differentiation of SLG1 confers indica rice to high-temperature tolerance, and tRNA thiolation pathway might be a potential target in the next generation rice breeding for the warming globe.

\footnotetext{
${ }^{1}$ State Key Laboratory of Plant Genomics, Institute of Genetics and Developmental Biology, the Innovative Academy of Seed Design, Chinese Academy of Sciences, Beijing 100101, China. ${ }^{2}$ College of Advanced Agricultural Sciences, University of Chinese Academy of Sciences, Beijing 100039, China.

${ }^{3}$ Department of Ecology, Evolution, and Organismal Biology, lowa State University, Ames, IA 50011, USA. ${ }^{4}$ These authors contributed equally: Yufang Xu,

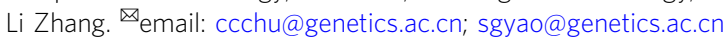


R ice (Oryza sativa L.) is an important food crop for over half of the world's population. The yield and quality of rice are often reduced by high-temperature stress ${ }^{1,2}$. Particularly, high temperature at the heading stage would cause premature degradation of anther tapetum, obstruction of pollen-tube elongation, abortion of pollen mother cells, decrease of pollen viability, and finally leading to reduction of spikelet fertility ${ }^{3-5}$. The high-temperature stress at the grain filling stage would result in poor milling quality, decreased grain weight and gel consistency, deteriorated starch structure, and increased chalkiness rate ${ }^{6,7}$. The damage of high-temperature stress to rice production would be further aggravated with climate change and global warming ${ }^{2}$.

Rice has adapted to different environmental conditions. The TT1 gene isolated from African rice (O. glaberrima Steud.) has been shown to contribute to the adaptation of rice plants under distinct climatic temperature ${ }^{8}$. For Asian cultivated rice, there are two genetically distinct subspecies, indica and japonica. indica rice is mainly distributed in tropical and subtropical regions, while japonica rice is mainly in temperate and cooler regions. Apparently, typical indica and japonica cultivars have been locally adapted to different environmental temperatures during natural and artificial selection ${ }^{9}$. For example, it has been shown that $C O L D 1^{j a p}$ and $b Z I P 73^{j a p}$, the selected natural variants during japonica domestication, can partially explain why japonica has stronger cold tolerance than indica ${ }^{10-12}$; distinct haplotypes of CTB4a have also been artificially selected and contributed to cold tolerance at the booting stage in japonica rice $^{13}$. However, the molecular basis underlying thermotolerance of indica rice is still poorly understood.

A recent analysis with the rice aet 1 mutant revealed the critical function of tRNA modification in the response to high temperature $^{14}$. As the indispensable participants in protein synthesis, tRNAs undergo abundant post-transcriptional modifications in both prokaryotes and eukaryotes, which are crucial for all the core aspects of tRNA functions including stress response ${ }^{15-17}$. As an important modification form, tRNA 2-thiolation refers to the thiolation on position $2\left(\mathrm{~s}^{2}\right)$ of the tRNA wobble uridine $\left(\mathrm{U}_{34}\right)$, which is often accompanied by the methoxycarbonyl methylation on position $5\left(\mathrm{mcm}^{5}\right)^{18,19} . \mathrm{mcm}^{5} \mathrm{~s}^{2} \mathrm{U}_{34}$ is exclusively found in three cytosolic tRNAs of $\mathrm{tK}^{\mathrm{UUU}}, \mathrm{tE}^{\mathrm{UUC}}$, and $\mathrm{tQ}^{\mathrm{UUG}}$, and the $\mathrm{s}^{2}$ modification in yeast (Saccharomyces cerevisiae) has been shown to depend on the URM1 pathway ${ }^{20-22}$. In this pathway, the Cterminal rhodanese-like domain of ubiquitin activating 4 enzyme (Uba4) provides sulfur to the ubiquitin-related modifier (Urm1), thereby forms a thiocarboxylated Urm1 (Urm1-COSH). And after the reductive cleavage of the acyl disulfide bond between Uba4 and Urm1, Urm1-COSH gets released to donate the activated sulfur for insertion into the target tRNA uridine facilitated by two cytosolic tRNA 2-thiolation proteins, Ncs6/CTU1 and $\mathrm{Ncs} 2 / \mathrm{CTU} 2^{20,22,23}$, which catalyze the last step of the thiolation process by forming a functional complex ${ }^{24}$.

A number of evidences showed that tRNA 2-thiolation in $\mathrm{U}_{34}$ has important roles in decoding and translation, and its defectiveness leads to various diseases in humans and pleotropic phenotypes in yeast ${ }^{21,25,26}$. In yeast, lack of the $U_{34}$ thiolation results in protein misfolding and aggregation ${ }^{27}$, inability to maintain metabolic homeostasis ${ }^{15,28}$, defects in invasive growth $^{29}$, and hypersensitivity to various abiotic stresses ${ }^{30,31}$. In particular, many studies suggested the importance of the $U_{34}$ thiolation in heat stress response. In yeast, the budding ability and propagation speed of the single and double mutants of ctu1/ctu2 are significantly lower than that of the wild-type strain under elevated temperature ${ }^{24}$. And, multicopy expression of tRNA ${ }^{\mathrm{LYS}}$, tRNA $^{\mathrm{GLU}}$, or a combination of both can rescue the growth defect of the ctu1 mutant strain ${ }^{24}$. Furthermore, dysfunction of the CTU1 homologs in archaea and Caenorhabditis elegans also causes thermosensitive phenotype ${ }^{24,32}$. Interestingly, a recent study compared the $\mathrm{U}_{34}$ thiolation profile from evolutionarily divergent yeast species following high-temperature stress, and revealed that thermotolerance and $s^{2} U_{34}$ level vary markedly among these yeast species. For example, the two commonly used laboratory strains (S288C and W303) of brewer's yeast Saccharomyces cerevisiae show temperature-dependent thiolation deficit that limits its growth to $39^{\circ} \mathrm{C}^{33}$. In contrast, in the haploid yeast Candida glabrata, which grows well at temperatures up to $43^{\circ} \mathrm{C}$, the thiolation level remains unaltered with the increase of temperature $^{33}$. Furthermore, a single-nucleotide polymorphism in Ncs2/CTU2 (i.e., H71L) results in a high-temperature growth phenotype ${ }^{34}$, and the thiolation level in ctu2 mutant of S. cerevisiae strain expressing $\mathrm{H} 71 \mathrm{~L}$ variant of CTU2 does not exhibit reduced tRNA thiolation at $37^{\circ} \mathrm{C}$ compared to that at $30^{\circ} \mathrm{C}^{35}$. Therefore, the $\mathrm{U}_{34}$ thiolation levels appear to be closely associated with thermotolerance in yeast, and the polymorphism in CTU2 allele may contribute to different $s^{2} \mathrm{U}_{34}$ levels and cell growth under elevated temperature. However, it is still unclear whether CTU2 has the conserved function in tRNA thiolation modification in rice. If so, whether the CTU2-mediated tRNA thiolation plays a role in rice response to high-temperature stress is unknown.

Here, we report the identification and characterization of SLG1 (Slender Guy 1) gene, which encodes the cytosolic tRNA 2thiolation protein 2 (RCTU2) and plays a key role in hightemperature tolerance in rice. We demonstrate that SLG1 is differentiated between indica and japonica rice, and the plants with the indica allele display enhanced thermotolerance. Our results suggest that artificial selection of SLG1 during domestication may have contributed to the adaptation of indica rice to hightemperature environment.

\section{Results}

Isolation of the $S L G 1$ gene. By screening a $\mathrm{NaN}_{3}$-mutagenized $\mathrm{M}_{2}$ library under the background of KY131 (temperate japonica, $T e j)$, we identified a mutant with slender plant architecture and increased grain length (Fig. 1a, b and Supplementary Fig. 1a, b, e) and named slg1 (for slender guy 1). Crossing of slg1 with WT yielded an $\mathrm{F}_{2}$ population, in which the segregation ratio of WT and longer grain phenotype was $3: 1\left(\chi^{2}=0.8<\chi_{0.05,1}^{2} ; 0.5>P>\right.$ 0.1 ; null hypothesis: fit 3:1). This result suggests that the $\operatorname{sg} 1$ mutant phenotype is due to the recessive mutation of a single locus. By using 20 bulked WT and mutant plants from the $\mathrm{F}_{2}$ population between $\operatorname{slg} 1$ and $\mathrm{KD} 8$ (temperate japonica), the candidate locus was directly mapped to the long arm of chromosome 12 between markers C1 and C5 (Fig. 1c). Further analysis of $834 \mathrm{~F}_{2}$ individuals with mutant phenotype narrowed down the target gene within a $310-\mathrm{kb}$ region between markers $\mathrm{C} 4$ and C6 (Fig. 1c). Sequence comparison revealed that there is a single-nucleotide substitution ( $G$ to $A$ ) at the splicing acceptor site of the fifth intron of LOC_Os12g39840 in the mutant (Fig. 1c and Supplementary Fig. 2a), resulting in mis-splicing and the formation of multiple transcripts (Supplementary Fig. 2b, c).

To confirm that LOC_Os12g39840 is indeed SLG1, the genomic DNA, including 1637-bp $5^{\prime}$ flanking sequence, SLG1 coding region, and 840-bp downstream sequence, was amplified from WT and transformed into slg1. All of the 21 independent $\mathrm{T}_{2}$ transgenic lines $(\operatorname{slg} 1-\mathrm{C})$ showed overall phenotypes resembling that of WT, including grain length and plant architecture (Fig. 1a, b). In addition, transgenic plants with specifically downregulated SLG1 expression under WT background (SLG1-RNAi) exhibited phenotypes similar to that of $\operatorname{slg} 1$ (Fig. 1a, b and Supplementary Fig. 3a), confirming that LOC_Os12g39840 is indeed the causal gene of the $\operatorname{sg} 1$ mutant. 
a

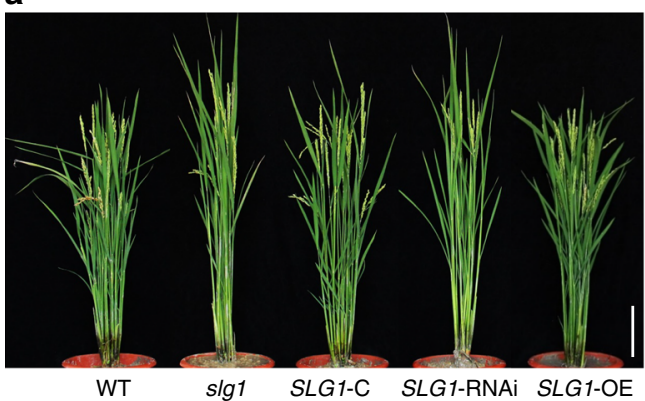

b

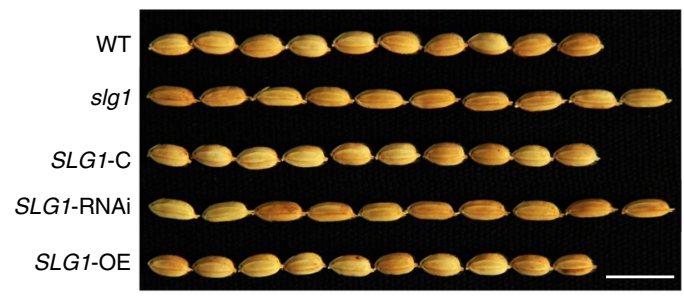

c

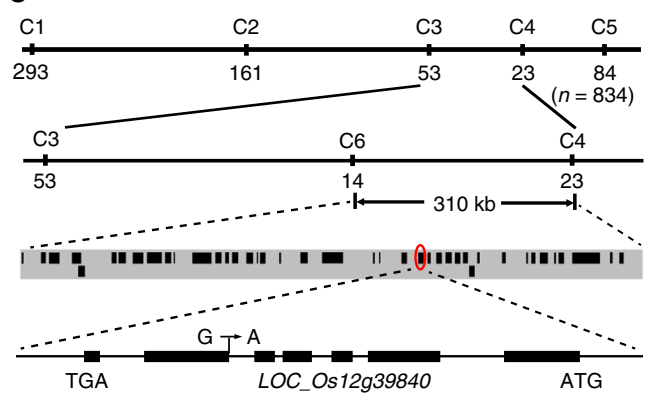

d

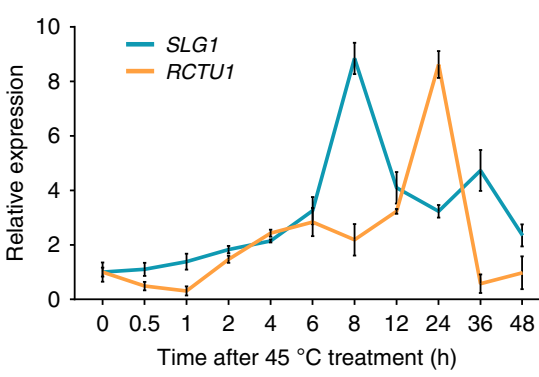

e

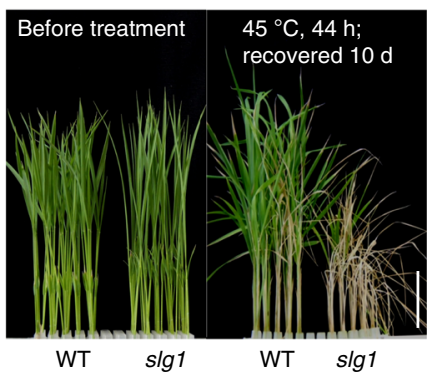

g
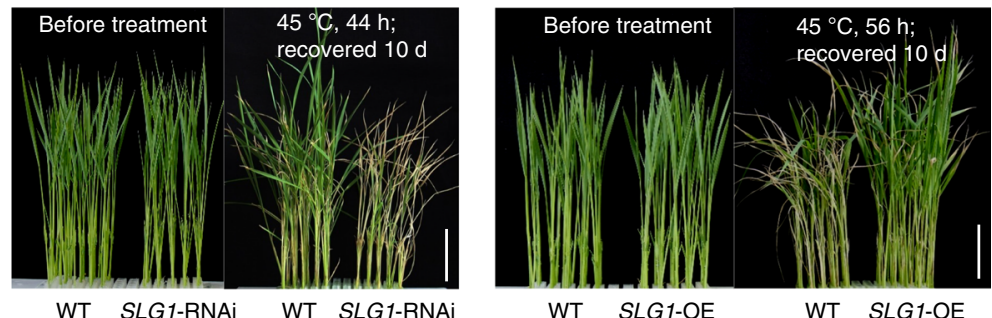

h

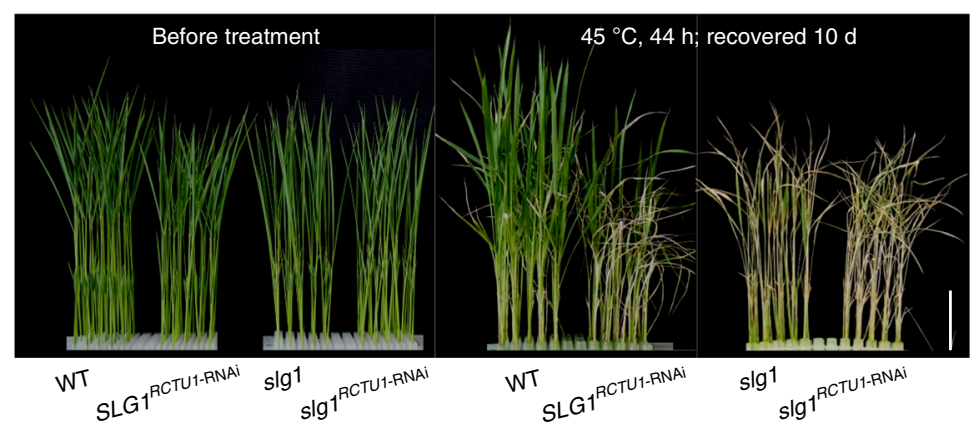

Fig. 1 SLG1 is a positive regulator of thermotolerance. a, b Phenotypes of whole plant and grain shape of slg1, WT, and the transgenic $T_{2}$ lines of slg1-C, SLG1-RNAi, and SLG1-OE. C, complementation; OE, overexpression; RNAi, RNA interference. Bars $=10 \mathrm{~cm}$ (a) and $1 \mathrm{~cm}$ (b). c Fine mapping of the SLG1 gene. A single-nucleotide substitution at the splicing acceptor site of the fifth intron of LOC_Os12g39840 in slg1 was indicated. d SLG1 and RCTU1 show hightemperature-inducible expression. Total RNA was extracted from the shoots of 2-leaf-stage seedlings at the indicated time-points. The expression levels were determined by $\mathrm{qPCR}$, and the expression level at time 0 was set to 1 . Actin was used as the internal control. Data are means \pm SD ( $n=3$ biological replicates). e-g SLG1 is a positive regulator of thermotolerance. Phenotypes of WT, slg1, SLG1-RNAi, and SLG1-OE plants before and after treatment are shown. Bars $=5 \mathrm{~cm}$. h Dysfunction of RCTU1 shows thermosensitive phenotype similar to the slg1 mutant. Phenotypes of RCTU1-RNAi plants on the background of $s / g 1$ and WT are shown before and after treatment. Bar $=5 \mathrm{~cm}$. The source data underlying (d) are provided as a Source data file.

SLG1 belongs to the CTU2 superfamily predicted to encode the cytoplasmic tRNA 2-thiolation protein 2 (Supplementary Fig. 4a), and the homologs of SLG1 were widely found in other organisms (Supplementary Fig. 5). Similar to the Arabidopsis counterpart of AtCTU2 ${ }^{36}$, SLG1 was found to be localized in both nucleus and cytoplasm (Supplementary Fig. 4b), and was ubiquitously expressed (Supplementary Fig. 4c). Moreover, loss-of-function of SLG1 and AtCTU2 causes similar defects in root morphology, such as reduced root length and lateral root density, and abnormal development of root hairs (Supplementary Fig. 1f-k) ${ }^{36}$.

SLG1 is a positive regulator of thermotolerance. One of the prominent biological functions of CTU1-CTU2 complex in yeast is the response to heat stress ${ }^{24,32-35}$. Expression of SLG1 was also significantly induced by high temperature (Fig. 1d). slg1 is highly thermosensitive, $\sim 84 \%$ of the mutant seedlings died after being treated at $45^{\circ} \mathrm{C}$ for $44 \mathrm{~h}$, while more than $90 \%$ of WT survived after recovery (Fig. 1e and Supplementary Fig. 3b). Similarly, the survival rate was also remarkably reduced for the SLG1-RNAi plants after high-temperature treatment (Fig. If and
Supplementary Fig. 3a, c). In contrast, the survival rate of SLG1OE seedlings was much higher $(>90 \%)$ than that of WT $(\approx 60 \%)$ under the same stress condition (Fig. 1g and Supplementary Fig. 3a, d), although the overall morphology of the SLG1-OE plants, such as grain length and plant architecture, was similar to that of WT under normal conditions (Fig. 1a, b). Taken together, these results indicate that $S L G 1$ functions positively in rice thermotolerance.

In yeast, CTU2 appears to catalyze the last step of tRNA thiolation process by forming a functional complex with CTU1 $1^{23,24,37}$. ROL5 is the homologous protein of Ncs6/CTU1 in Arabidopsis ${ }^{38}$. Therefore, we used the amino-acid sequence of ROL5/AtCTU1 as the query to search for the homologous proteins in the rice genome. We identified two homologous proteins of LOC_Os02g52470 and LOC_Os01g41565 in rice. LOC_Os02g52470 shares the highest sequence similarity with ROL5/AtCTU1 and is annotated as the rice CTU1 protein (RCTU1). We then conducted the interaction assays to test the presence of CTU1-CTU2 complex both in vivo and in vitro and observed a clear interaction between RCTU1 and SLG1 
a
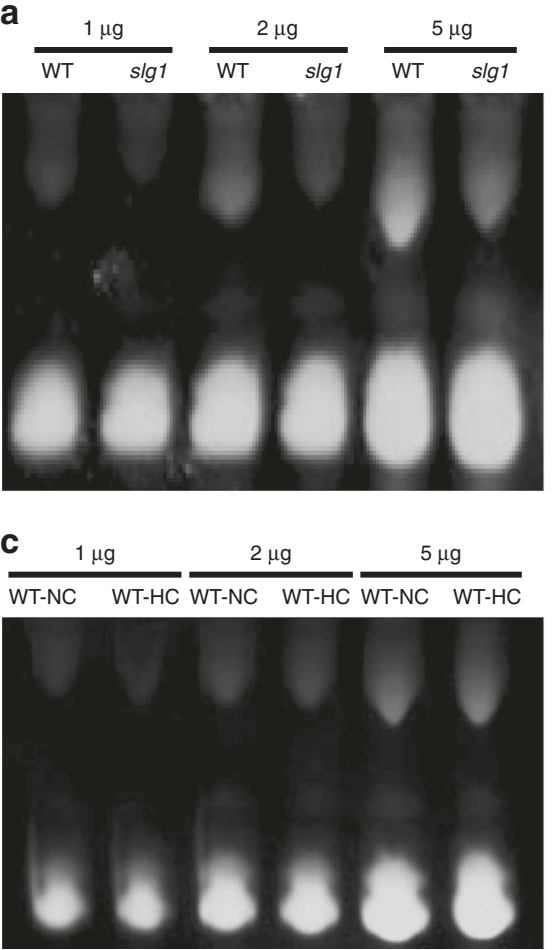

$\frac{\mathrm{tK}^{\mathrm{UUU}}}{\mathrm{WT} \quad \operatorname{slg} 1} \frac{\mathrm{tE}^{\mathrm{UUC}}}{\mathrm{WT} \quad \operatorname{slg} 1} \frac{\mathrm{tQ}^{\mathrm{UUG}}}{\mathrm{WT} \quad s \operatorname{si} 1}$
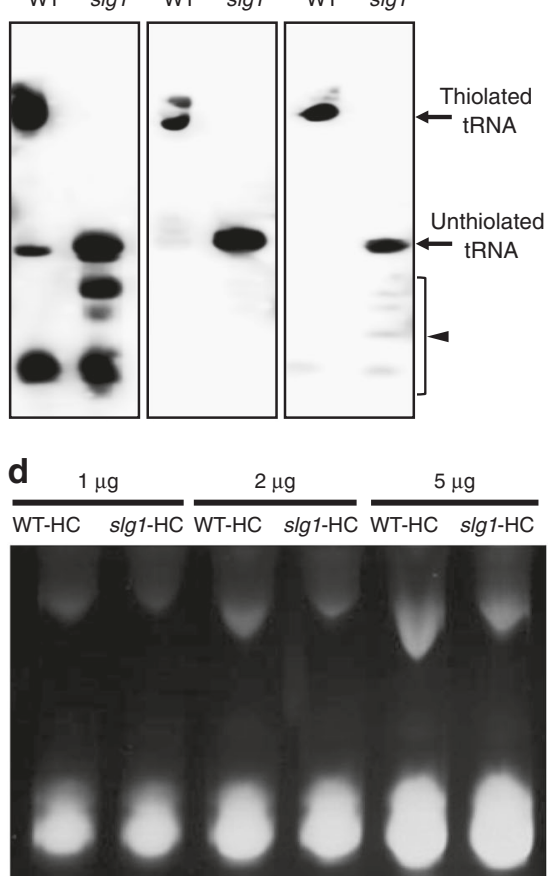

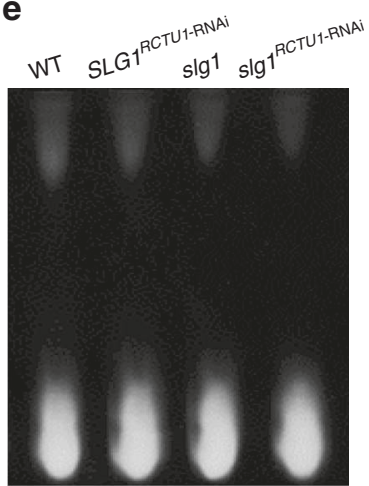

$\mathbf{f}$
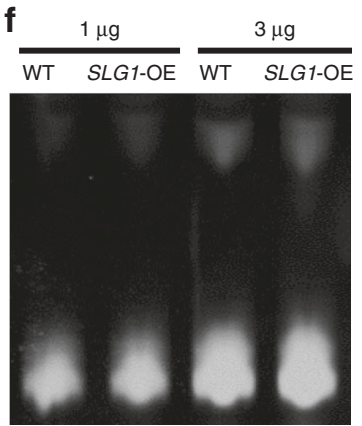

Fig. 2 SLG1-mediated thermotolerance is positively correlated with thiolated tRNA level. a Comparison of thiolated tRNA level between slg1 and WT. Shoots of 2-leaf-stage seedlings under normal conditions were sampled. The slow-migration band indicates thiolated tRNAs in the presence of APM. The loaded amount of tRNA was 1, 2, and $5 \mu \mathrm{g}$ for each lane, respectively. b Northern blot analysis. The loaded amount of tRNA was $1 \mu \mathrm{g}$, which was extracted from 2-leaf-stage normal-grown seedlings. Three tRNA isoacceptors of $t K \cup U U, t E \cup U C$, and $t Q U U G$ were used to detect the specifically thiolated tRNAs. The bands observed in tK (arrowhead) might be indicative of tRNA halves or tRNA degradation. c tRNA thiolation process was adversely affected by hightemperature stress. Shoots of 2-leaf-stage seedlings grown under high-temperature $\left(45^{\circ} \mathrm{C}, 44 \mathrm{~h}\right)$ and normal conditions were sampled, respectively. The loaded amount of tRNA was 1, 2, and $5 \mu \mathrm{g}$ for each lane, respectively. NC, normal conditions; $\mathrm{HC}$, high-temperature conditions. $\mathbf{d}$-f $\mathrm{C}$ Comparison of thiolated tRNA level. Shoots of 2-leaf-stage seedlings under high-temperature $\left(45^{\circ} \mathrm{C}, 44 \mathbf{h} ; \mathbf{g}\right)$ or normal conditions (h, i) were sampled, respectively. The loaded amount of tRNA was 1, 2, and $5 \mu \mathrm{g}(\mathbf{d}), 3 \mu \mathrm{g}(\mathbf{e}), 1$ and $3 \mu \mathrm{g}(\mathbf{f})$ for each lane, respectively. The experiments in (a-f) were repeated three times with similar results. Source data are provided as a Source data file.

(Supplementary Fig. 6a-c). To study if RCTU1 works in the same genetic pathway as SLG1, we constructed RCTU1-RNAi transgenic plants under the background of WT (SLG1 $\left.{ }^{R C T U 1-R N A i}\right)$ and slg1 $\left(\operatorname{sg} 1^{R C T U 1-\mathrm{RNAi})}\right.$, respectively. We found that both

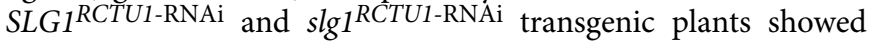
similar phenotypes to $\operatorname{sg} 1$, including increased grain length, slender plant architecture, and defective root system (Supplementary Figs. 1 and 6d, e). Moreover, similar to SLG1, transcription of RCTU1 was also highly induced by high temperature (Fig. 1d), and the survival rates of SLG1 ${ }^{R C T U 1-R N A i}$ and $\operatorname{sg} 1^{R C T U 1-R N A i}$ seedlings were all greatly reduced under high temperature (Fig. $1 \mathrm{~h}$ and Supplementary Fig. 3e). These results indicate that the RCTU1-SLG1 complex plays an essential role in rice thermotolerance.

SLG1-mediated thermotolerance is positively correlated with thiolated tRNA levels. It was reported that the CTU1-CTU2 complex is responsible for the 2-thiolation of cytosolic tRNAs, and the loss of thiolation results in the thermosensitive phenotype in the CTU2- and CTU1-deleted yeast strains ${ }^{24}$. To characterize the role of tRNA thiolation in high-temperature stress response, we first compared the thiolated tRNA levels between slg1 and WT using a polyacrylamide gel supplemented with APM (N-acryloylamino phenyl mercuric chloride). Thiolated tRNAs can be specifically retarded by APM due to the affinity of the thio group with the mercuric compounds ${ }^{36}$. We found that the amount of thiolated tRNAs in slg1 was much lower than that in WT
(Fig. 2a), suggesting that the loss-of-function of SLG1 impaired tRNA thiolation in the mutant. The defective tRNA thiolation in slg1 was further confirmed by Northern blot. We found that dysfunction of SLG1 specifically impaired the thiolation level of $\mathrm{tK}^{\mathrm{UUU}}$, $\mathrm{tE}^{\mathrm{UUC}}$, and $\mathrm{tQ}^{\mathrm{UUG}}$ (Fig. 2b), which was similarly observed for Ncs2/CTU2 in yeast. Next, we examined the effect of high temperature on tRNA thiolation by comparing the thiolated tRNA levels under both normal and high-temperature conditions. It was found that elevated temperature influenced the thiolation of $\mathrm{U}_{34}$ and caused tRNA hypothiolation in rice (Fig. 2c). The thiolated tRNA levels in WT were much higher than that in $\operatorname{slg} 1$ under high temperature (Fig. 2d), which is consistent with the higher survival rate of WT (Fig. 1e and Supplementary Fig. 3b). With the decreasing level of thiolated tRNAs in the WT, $S L G 1^{R C T U 1-R N A i}$, slg1, and $\operatorname{sg} 1^{R C T U 1-R N A i}$ (Fig. 2e), the survival rates of plants were reduced to $90 \%, 36.5 \%, 15.2 \%$, and $5 \%$, respectively (Fig. $1 \mathrm{~h}$ and Supplementary Fig. 3e). In contrast, the SLG1-OE plants with increased thiolated tRNA levels conferred enhanced thermotolerance compared with WT (Figs. 1g, 2f, and Supplementary Fig. 3d). Taken together, these results suggest that the SLG1-mediated thermotolerance is positively correlated with the thiolated tRNA levels in vivo.

SLG1 may have undergone selective sweep in Asian cultivated rice. In Asian cultivated rice, the two subspecies indica and japonica are distributed in distinct ecological regions and possess contrasting phenotype under high-temperature stress ${ }^{39}$ 


\begin{tabular}{|c|c|c|c|c|c|c|c|c|}
\hline \\
\hline Position
\end{tabular}
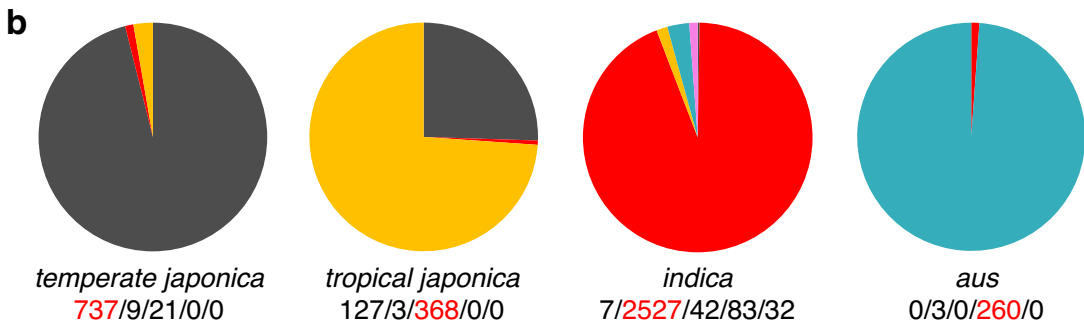

- Hap1

- Hap2

Hap3

- Hap4

Hap5

Fig. 3 SLG1 is differentiated in Asian cultivated rice. a Haplotype analysis of SLG1 promoter and coding regions from 4219 Asian cultivated rice accessions. Red letters indicate different nucleotides, and gray-marked letters indicate non-synonymous substitutions. b Distribution frequency of the five SLG1 haplotypes in diverse Asian cultivated rice collection. The number of cultivars for each haplotype was given from left to right below each subpopulation. The haplotype with the largest number was highlighted in red. The source data underlying (b) are provided as a Source data file.

(Supplementary Fig. 7a-c). Our results above clearly demonstrated that the thermotolerance of rice is closely related to SLG1mediated tRNA 2-thiolation. A sequence comparison revealed that both the promoter and coding regions of SLG1 in japonica cultivars were different from that in indica (Ind) cultivars (Supplementary Fig. 8). We further analyzed natural variations of SLG1 using 4219 sequenced rice accessions ${ }^{40}$, and found ten and seven SNPs in the promoter and coding regions of SLG1, respectively (Fig. 3a). Of the seven SNPs in the coding region, the two nucleotide substitutions of T865C and G1839T caused amino-acid conversions from $\mathrm{Val}^{177}$ to $\mathrm{Ala}^{177}$ and $\mathrm{Val}^{362}$ to $\mathrm{Phe}^{362}$, respectively, while the other five were synonymous substitutions (Fig. 3a). The two amino-acid mutations were found in cultivated rice, with $98.2 \%$ of indica containing $\mathrm{Val}^{177}-\mathrm{Val}^{362}$ and 99.1\% of japonica possessing $\mathrm{Ala}^{177}-\mathrm{Phe}^{362}$, suggesting their contributions to the functional divergence of SLG1.

Based on the polymorphisms, the 4219 accessions could be classified into five haplotypes (Fig. 3b), with 737 out of 767 (96.1\%) temperate japonica accessions carried Hap1, while 2527 out of 2691 (93.9\%) indica accessions carried Hap2. Notably, almost all the aus accessions (98.9\%) could be distinctly classified as Hap4. The tropical japonica (Trj) group has two major haplotypes, Hap3 (73.9\%) and Hap1 (25.5\%), which only differ in a single nucleotide (A252C) in the promoter region. Hap5, found only in the indica group at a very low frequency $\left({ }^{\circ} 0.8 \%\right)$, was highly similar to Hap2 except a single nucleotide difference (G432A) in the promoter region. We further compared the nucleotide sequence of Hap1-Hap4 with 34 O. rufipogon wild rice accessions (Supplementary Data 1). Beside the abundant variations across the SLG1 genomic region among wild rice accessions, a number of SLG1 haplotypes in wild rice accessions were exactly the same as that of Ind, Tej, Trj, and Aus. For example, the SLG1 allele of the indica cultivar 93-11 is identical with that of the wild rice accession W1687 (Hap2); the aus cultivar Kasalath shares the same haplotype with W593 (Hap4); the temperate japonica cultivar Nipponbare carries the same SLG1 allele as W3078 (Hap1), while the tropical japonica cultivar NERICA3 has the same allele as W120 (Hap3). These results imply that $S L G 1$ Tej, $S L G 1^{T r j}, S L G 1^{I n d}$, and $S L G 1^{A u s}$ might represent an ancient allele preserved in different $O$. rufipogon wild rice accessions, and might have been subjected to positive selection at early stages of rice domestication.

Regional reduction of nucleotide diversity $(\pi)$ indicates positive selection ${ }^{11}$. We estimated the nucleotide diversity across the SLG1 
genomic region in the four rice subpopulations. Compared with O. rufipogon $(\pi=0.004098$; Supplementary Fig. 9 and Supplementary Table 1), the immediate ancestral progenitor of cultivated rice, the nucleotide diversity of SLG1 was extremely low in all four cultivated rice subpopulations of indica $(\pi=$ $0.000098)$, temperate japonica $(\pi=0.000073)$, tropical japonica $(\pi=0.000541)$, and Aus $(\pi=0.000073)$, which was also much lower than that in the whole genome of $O$. sativa $(\pi=0.0024)^{41}$. We then measured the ratio of the genetic diversity in wild rice to that in cultivated rice $\left(\pi_{\mathrm{w}} / \pi_{\mathrm{c}}\right)$ across the SLG1 region, and found the $\pi_{\mathrm{w}} / \pi_{\mathrm{c}}$ in all four subpopulations $\left(\pi_{\mathrm{w}} / \pi_{\mathrm{c}}=7-55\right)$ was much higher than the top 5\% genome-wide threshold used as the selection cutoff $\left(\pi_{\mathrm{w}} / \pi_{\mathrm{c}}=3 \text {; Supplementary Table } 1\right)^{41}$, suggesting that SLG1 was subjected to strong selection in all these subpopulations. Because positive selection may lead to a selective sweep in the flanking region of the selected gene ${ }^{11}$, we further examined the nucleotide diversity of $30-\mathrm{kb}$ regions flanking SLG1. As expected, the average nucleotide diversity of SLG1 flanking regions in temperate japonica $(\pi=0.000173)$, tropical japonica $(\pi$ $=0.000542)$, indica $(\pi=0.000972)$, and Aus $(\pi=0.000634)$ were comparable to that of the $S L G 1$ region but much lower than that in the corresponding wild rice populations $(\pi>0.0033$; Supplementary Table 1). Further, we identified MB-sized low diversity region in both indica and temperate japonica populations spanning the SLG1 locus (Supplementary Fig. 9), which is consistent with the selective sweep argument. Regardless, the bottleneck effect may also have its contribution to the diversity signal we have been observing, giving the bottleneck in japonica is much intense ${ }^{41}$, which is also revealed by our data (Supplementary Table 1).

To further explore the evolutionary signature of SLG1 in indica and japonica subpopulations, we constructed a neighbor-joining tree of SLG1 and compared to that of random SNPs. Both trees displayed indica-japonica segregation and the SLG1 haplotype of admixture samples also displayed such segregation (Supplementary Fig. 10), suggesting the lack of introgression in SLG1. Further, we calculated the fixation index $\left(F_{\mathrm{ST}}\right)$ around $S L G 1$ among different populations, and observed a high level of differentiation between the indica and temperate japonica populations, confirming the lack of introgression between the two populations (Supplementary Fig. 11). To be noted, the selective sweep region (Supplementary Fig. 9) contains over 500 genes including SLG1, any of which could be the driving factor of the selective sweep. Nevertheless, these data suggested that the differentiation of SLG1 was driven by selection acting on this region.

SLG1 ${ }^{\text {Ind }}$-carrying plants show strong thermotolerance. The USDA Mini-Core germplasms are a diverse collection of landraces that were not derived from modern breeding ${ }^{42}$. Through mapping the geological distribution of SLG1 haplotypes in MiniCore germplasms alongside with the 30-year (1960-1990) average terrestrial temperature, we found that Hap2, the allele carried by 93.9\% indica accessions, is predominantly distributed in the regions with annual mean temperature above $29^{\circ} \mathrm{C}$. In contrast, Hap1, the allele carried by $96.1 \%$ temperate japonica accessions, is predominant in the regions with annual mean temperature below $18^{\circ} \mathrm{C}$ (Supplementary Fig. 12a). And the similar geographic distribution was observed when plotting the SLG1-Hap1/Hap2 distribution in admixture germplasms alongside with the average terrestrial temperature (Supplementary Fig. 12b). These results suggest that the Hap1 and Hap2 haplotypes of SLG1 might have potential contribution for different environmental-temperature adaptation of rice.

In view of the fact that indica and japonica rice have experienced clear adaptation to distinct environmental temperature $9,39,41,43$, and the important role of SLG1 in thermotolerance, we speculate that the differentiation of SLG1 between indica and japonica might be one of the decisive factors for the two subspecies to tolerate different environmental temperature. Therefore, we constructed nearisogenic lines (NIL) by introducing the indica allele of SLG1 $\left(S L G 1^{\text {Ind }}\right)$ into the background of KY131 (SLG1 $\left.1^{T e j}\right)$, and vice versa (Fig. 4a). The obtained NIL-SLG1 $1^{\text {Ind }}$ and NIL-SLG1 $1^{\text {Tej }}$ plants exhibited the same appearance as their corresponding recipients at seedling stage under normal growth conditions (Fig. 4b). When encountering high-temperature stress, the survival rate of NIL$S L G 1^{T e j}$ seedlings was much lower $(50 \%)$ than that of the indica recipient ZF802 (93\%) (Fig. 4b). In contrast, the NIL-SLG1 Ind seedlings exhibited markedly enhanced thermotolerance relative to KY131, with the mean survival rate of about $81 \%$ in NIL-SLG1 Ind compared with that of about $48 \%$ in KY131 (Fig. 4b), indicating that $S L G 1^{I n d}$ is superior to $S L G 1^{T e j}$ in plant tolerance to hightemperature stress.

To further evaluate the functional difference between SLGI Ind and $S L G 1^{T e j}$, we adopted transgenic approach by amplifying the promoter and gene sequences of SLG1 from KY131 and 93-11, and introduced them into $s l g 1$ to generate complementary plants JPJC (the Tej-type promoter with Tej-type gene sequences) and IPIC (the Ind-type promoter with Ind-type gene sequences), respectively (Supplementary Fig. 13a). We planted 32 plants for each transgenic line and observed overall agronomic traits including plant height, heading date, grain length, grain width, and seed-setting rate under normal growth conditions in three growing seasons, and three independent $\mathrm{T}_{3}$ transgenic lines with WT-phenotype were selected for thermotolerance comparison. We found that the IPIC lines exhibited much higher thermotolerance than that of JPJC, with the mean survival rate of about $69 \%$ in IPIC compared with that of about $29 \%$ in JPJC (Fig. 5a, b). The enhanced thermotolerance conferred by SLG1 Ind was in accordance with the better adaptability of indica to the tropical or subtropical regions. Notably, the overall gross morphology of the IPIC plants including grain length and plant architecture was quite similar to that of WT and JPJC plants under normal growth conditions (Supplementary Figs. 13b, c and 14), suggesting that the $S L G 1^{\text {Ind }}$ allele has great potential in thermotolerance breeding.

To understand the role of tRNA thiolation in differential thermotolerance of $S L G 1^{\text {Ind }}$ and $S L G 1^{T e j}$, we quantified the thiolated tRNAs in IPIC and JPJC plants by liquid chromatography-tandem quadrupole mass spectrometry (LC/ MS-QQQ) using synthetic $\mathrm{mcm}^{5} \mathrm{~s}^{2} \mathrm{U}$ as the standard. Similar to the results of APM-PAGE analysis (Fig. 2a), the thiolated tRNA level in slg1 was markedly decreased compared to that in WT (Fig. 5c), and introduction of either SLG1 ${ }^{\text {Ind }}$ or $S L G 1^{T e j}$ rescued the thiolation defect of the mutant (Fig. 5c). Notably, we found that the $\mathrm{mcm}^{5} \mathrm{~s}^{2} \mathrm{U}$ amounts in IPIC were much higher than that in JPJC (Fig. 5c), which was also observed by the APM-PAGE method (Fig. 5d). Taken together, these results strongly suggest that SLG1-mediated tRNA thiolation is responsible for the difference in thermotolerance between $S L G 1^{\text {Ind }}$ and $S L G 1^{T e j}$.

The variations at both promoter and coding sequences of SLG1 contribute to thermotolerance. In addition to the ten SNPs found in the 1637-bp promoter region of SLG1, seven InDels were also found in this region (Supplementary Fig. 8). It was unclear whether the polymorphism of the promoter region or the conversion of two amino acids in coding region was responsible for the differential thermotolerance between $S L G 1^{T e j}$ and $S L G 1^{\text {Ind }}$. Although the $1637-b p$ promoter of SLG1 was sufficient for complementary test (Supplementary Fig. 13), the luciferase activity of $p S L G 1-L U C$ was extremely low in both constructs of 


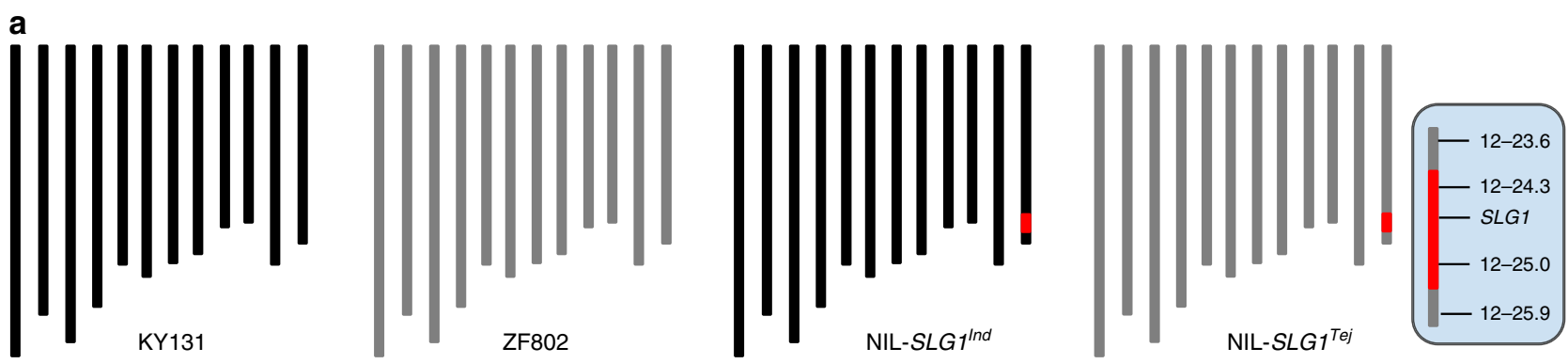

b
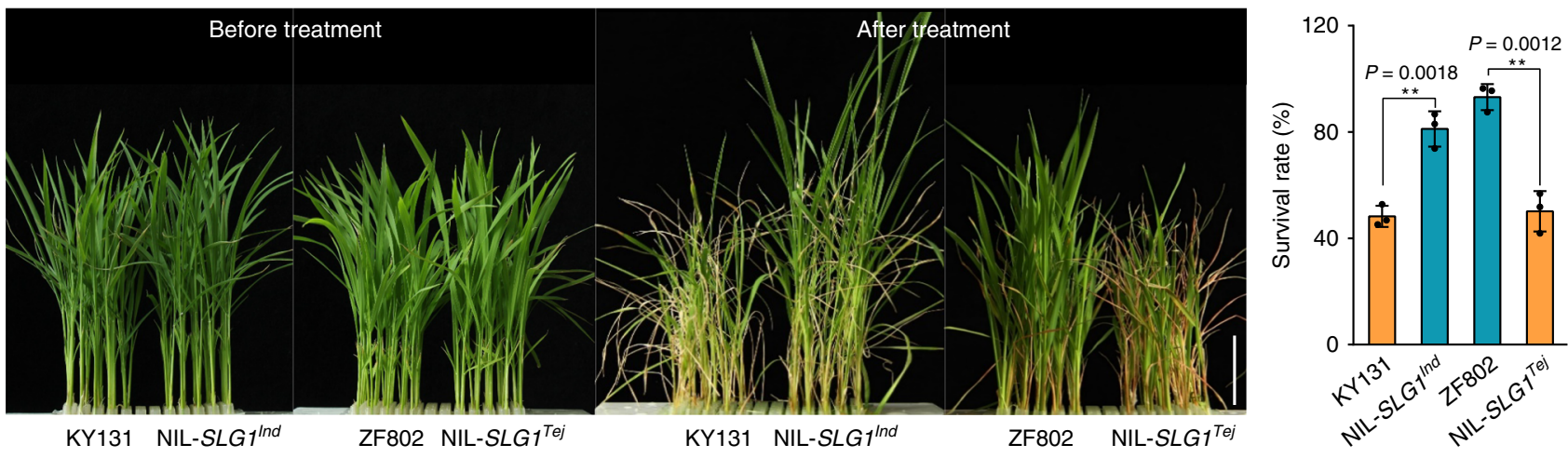

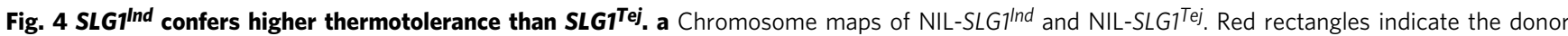
segment containing the SLG1 locus. NIL-SLGIInd indicates KY131 (temperate japonica) with SLGIInd allele from ZF802 (indica), and NIL-SLG1Tej indicates ZF802 with SLGT Tej allele from KY131. b Comparison of thermotolerance between SLGIInd and SLGT Tej alleles. Two-leaf-stage seedlings were treated with $45^{\circ} \mathrm{C}$ for $56 \mathrm{~h}$ for $\mathrm{KY} 131$ and NIL-SLGIInd , and $64 \mathrm{~h}$ for ZF802 and NIL-SLG1Tej, respectively. The seedlings were then recovered under normal conditions for 10 days. Data are means \pm SD ( $n=3$ biological replicates, with $80-96$ seedlings per biological replicate). ${ }^{\star \star} P<0.01$, two-tailed Student's $t$-test. Bar $=5 \mathrm{~cm}$. The source data underlying (b) are provided as a Source data file.

SLG1 Tej and SLG1 Ind in vitro (Supplementary Fig. 15a). We speculate that other regulatory elements may need for SLG1 transcription. Therefore, the activity of the two promoters of $S L G 1^{I n d}$ and $S L G 1^{T e j}$ in protoplasts may not reflect the situation in vivo. In contrast, the in vivo transcription of SLG1 was significantly higher in indica cultivars even under normal growth conditions $(P=0.0016$, two-tailed $t$-test; Supplementary Fig. $7 \mathrm{~d})$, and higher level of SLG1 expression in NIL-SLG1 ${ }^{\text {Ind }}$ (KY131 background) and IPIC plants was also observed under either normal condition or heat stress compared with that in the recipient KY131 and JPJC plants, respectively (Supplementary Fig. 15b-d). Together with the thermosensitivity of SLG1-RNAi plants and the thermotolerance of SLG1-OE plants (Fig. 1f, g), these results clearly demonstrate the positive correlation between SLG1 transcription level and thermotolerance.

To explore whether the polymorphism of promoter regions results in the difference of SLG1 expression, we developed two promoter-swapping vectors JPIC (the Tej-type promoter with Indtype gene sequences) and IPJC (the Ind-type promoter with Tejtype gene sequences) using the 1637-bp promoter and gene sequences of $S L G 1$, and were then transformed into slg1 mutant, respectively (Supplementary Fig. 13a). Under normal growth conditions, the gross morphology and grain shape among IPIC, IPJC, JPIC, and JPJC plants were comparable to each other (Supplementary Fig. 13b, c). When subjecting the four complementary seedlings to high-temperature stress, we found that the survival rate of IPIC plants was the highest (69\%), while that of JPJC plants was the lowest (29\%). Interestingly, the survival rates of IPJC plants (49\%) and JPIC plants (47\%) were significantly higher than that of JPJC plants but lower than that of IPIC plants $(P<0.05$, two-tailed $t$-test), while no significant difference was observed between IPJC plants and JPIC plants $(P=0.8160$, two- tailed $t$-test) (Fig. 5a, b). These results indicate that the variations at both promoter and coding regions collectively contribute to the SLG1-mediated thermotolerance.

SLG1 ${ }^{\text {Ind }}$ enhances thermotolerance at the reproductive stage. Rice plant is highly sensitive to high-temperature stress especially at the reproductive stage ${ }^{44}$, and improving thermotolerance at the reproductive stage is thus of particular importance to reduce yield loss. Therefore, we performed high-temperature treatment of the adult plants of slg1 and WT to evaluate the role of SLG1 at the reproductive stage. We found that the seed-setting rate of slg1 was less than $28 \%$, while that of WT could reach to $72 \%$ after treatment at $40{ }^{\circ} \mathrm{C}$ for five days (Fig. $6 \mathrm{a}, \mathrm{b}$ ), suggesting that the slg1 mutant was also sensitive to high-temperature stress at the reproductive stage. Microscopic examination found that the pollen viability of $s l g 1$ was moderately reduced compared with that of WT under the stress condition (Supplementary Fig. 16a), but the number of pollen grains on the stigma of slg1 was significantly reduced (Supplementary Fig. 16b, c), and pollen-tube elongation could be hardly observed in the slg1 mutant under high-temperature treatment (Supplementary Fig. 16d). These results demonstrated that $S L G 1$ plays an important role in thermotolerance at the reproductive stage.

In our NIL lines, we observed a decreased fertility for NILSLG1 Ind plants (Supplementary Fig. 17), which is likely due to the tight linkage of SLG1 with the hybrid sterility gene $H S A 1^{45}$. To further explore the possible role of $S L G 1$ at the reproductive stage, we selected eight independent lines from each of the IPIC, IPJC, JPIC, and JPJC constructs, half of which were treated with high temperature for 5 days and the other half for 7 days at the microsporocyte meiosis stage, respectively. Among the four 

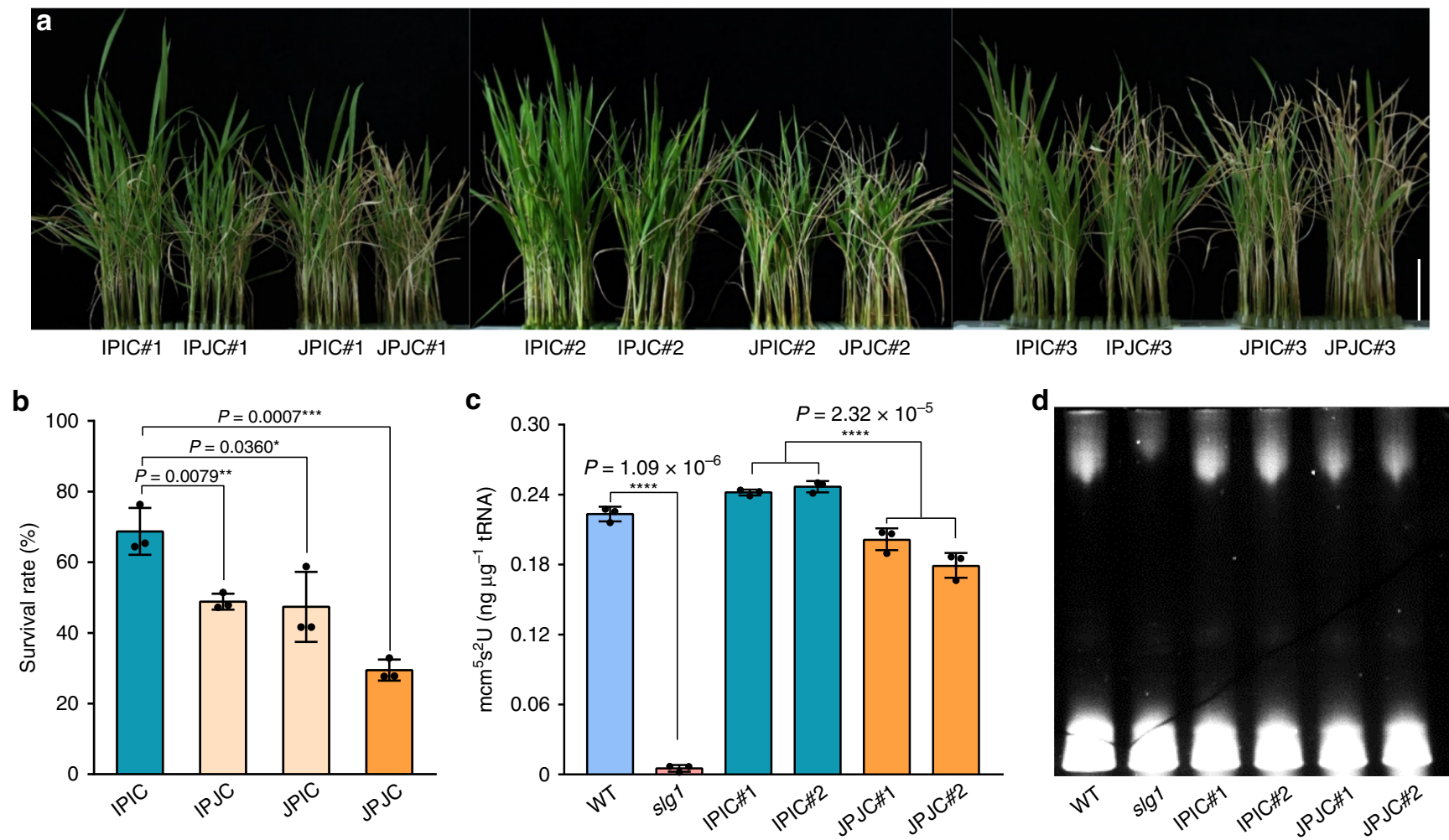

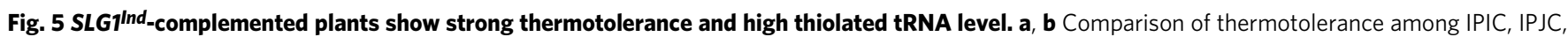
JPIC, and JPJC plants. Three independent homozygous IPIC, IPJC, JPIC, and JPJC lines were selected and treated with $45^{\circ} \mathrm{C}$ for $56 \mathrm{~h}$ and then recovered under normal condition for 10 days. Data are means \pm SD $(n=3$ independent transgenic lines. Each transgenic line was analyzed with three biological

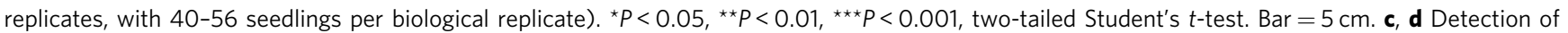
thiolated tRNAs in slg1, WT, IPIC, and JPJC transgenic lines based on LC/MS-QQQ- (c) and APM- (d) methods. Shoots of 2-leaf-stage seedlings under normal growth conditions were sampled. The loaded amount of tRNA was $3 \mu \mathrm{g}$ for each lane (d). Data are means \pm SD ( $n=3$ biological replicates). ${ }^{\star \star \star \star} P<$ 0.0001, two-tailed Student's t-test. The experiment in (d) was repeated three times with similar results. The source data underlying (b-d) are provided as a Source data file.

complementary lines, the IPIC lines showed the strongest thermotolerance in terms of seed-setting rate, which is about $81 \%$ and $49 \%$ after 5 and 7 days high-temperature treatment, respectively (Fig. 6c, d and Supplementary Fig. 18). In contrast, the seed-setting rate of JPJC was only about $47 \%$ and $22 \%$, respectively, under the same stress condition (Fig. $6 \mathrm{c}$, d and Supplementary Fig. 18). Again, we could not distinguish the function of the promoter from that of the coding sequence, because there was no significant difference in the seed-setting rate between IPJC and JPIC lines $(P>0.30$, two-tailed $t$-test). The seed-setting rates of IPJC lines after 5 and 7 days of treatment were about $68 \%$ and $43 \%$, respectively, while those of JPIC lines were about $63 \%$ and $37 \%$ (Fig. $6 \mathrm{c}$, d and Supplementary Fig. 18). These results further suggest that variations at both promoter and coding sequences of SLG $1^{\text {Ind }}$ contribute to the thermotolerance in rice.

\section{Discussion}

With global warming and climate change, understanding how plants respond to high-temperature stress and breeding thermotolerant crops are urgently needed for sustainable agriculture. However, few thermotolerance-related genes have so far been identified and characterized. In this study, we provided several lines of evidence that tRNA thiolation plays an important role in rice thermotolerance. First, SLG1 and RCTU1, the rice homologous genes of yeast Ncs2/CTU2 and Ncs6/CTU1, respectively, were strongly induced by high temperature (Fig. 1d). Second, dysfunction of SLG1 resulted in a significant decrease in the thiolated tRNA level in vivo (Figs. $2 \mathrm{a}, \mathrm{b}$ and $5 \mathrm{c}$ ), and the corresponding mutant displayed thermosensitive phenotype (Fig. 1e and Supplementary Fig. 3b). Third, the survival rates of WT, $S L G 1^{R C T U 1-R N A i}, \operatorname{slg} 1$, and $s l g 1^{R C T U 1-R N A i}$ decreased after hightemperature treatment (Fig. $1 \mathrm{~h}$ and Supplementary Fig. 3e), which were consistent well with the decreasing level of thiolated tRNAs in these plants (Fig. 2e). Fourth, overexpression of SLG1 increased the thiolated tRNA level and enhanced the thermotolerance of the transgenic plants (Figs. 1g, 2f, and Supplementary Fig. 3d). Fifth, the thiolated tRNA level of the complementary IPIC plants was higher than that of the JPJC plants (Fig. $5 \mathrm{c}, \mathrm{d}$ ), and the thermotolerance of the former was also much better than that of the latter (Figs. $5 \mathrm{a}, \mathrm{b}$ and $6 \mathrm{c}, \mathrm{d}$ ). In addition, the complementary lines of IPIC and JPJC displayed differences in thiolated tRNA level and thermotolerance (Figs. 5 and $6 c$, d), although their morphological traits were similar to WT under normal growth conditions (Supplementary Figs. 13 and 14). Therefore, our results revealed the importance of SLG1-mediated tRNA thiolation in rice thermotolerance.

Numerous studies have revealed that tRNA thiolation of $\mathrm{U}_{34}$ plays a critical role in fine-tuning protein translation. Lacking of $\mathrm{U}_{34}$ thiolation in $\mathrm{tK}^{\mathrm{UUU}}, \mathrm{tE}^{\mathrm{UUC}}$, and $\mathrm{tQ}^{\mathrm{UUG}}$ leads to ribosome pausing at their cognate codons, thus influencing the rate of translation and protein folding, and causing proteotoxic stress and endogenous protein aggregation in yeast ${ }^{27,46,47}$. Further, we explored the effects of tRNA thiolation on proteome, and found that dysfunction of SLG1 affects the accumulation of plenty of 
a

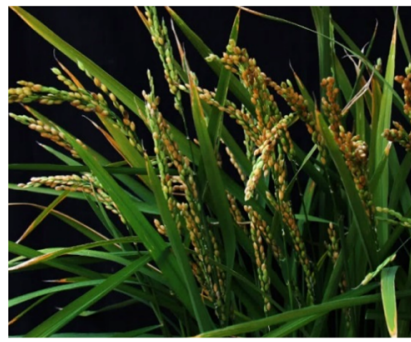

WT

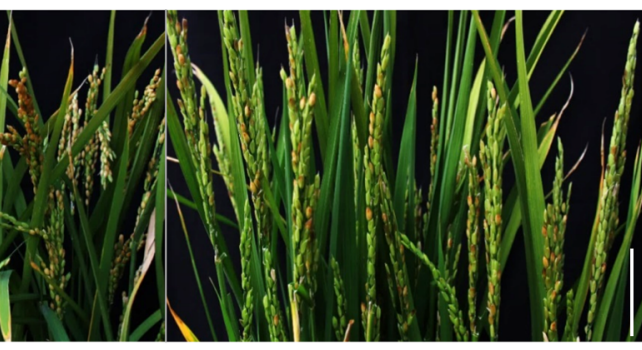

$\operatorname{slg} 1$

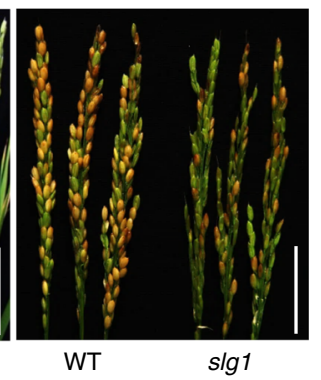

b

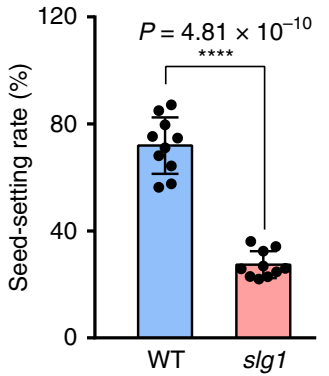

C

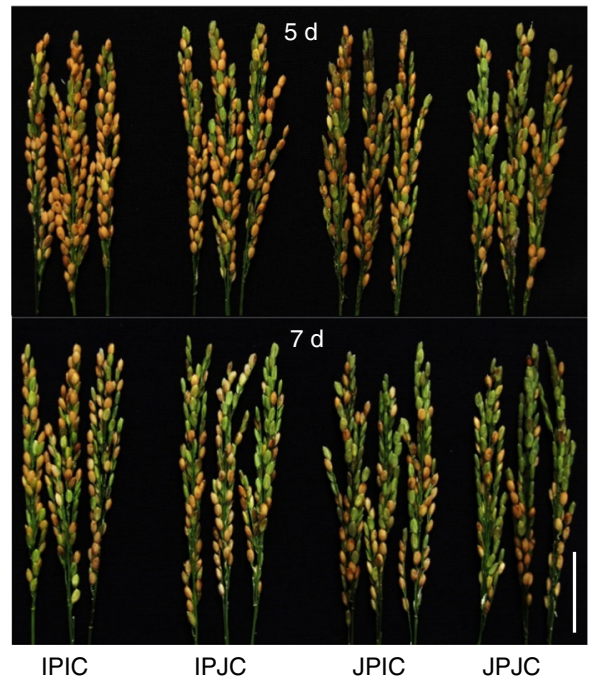

d

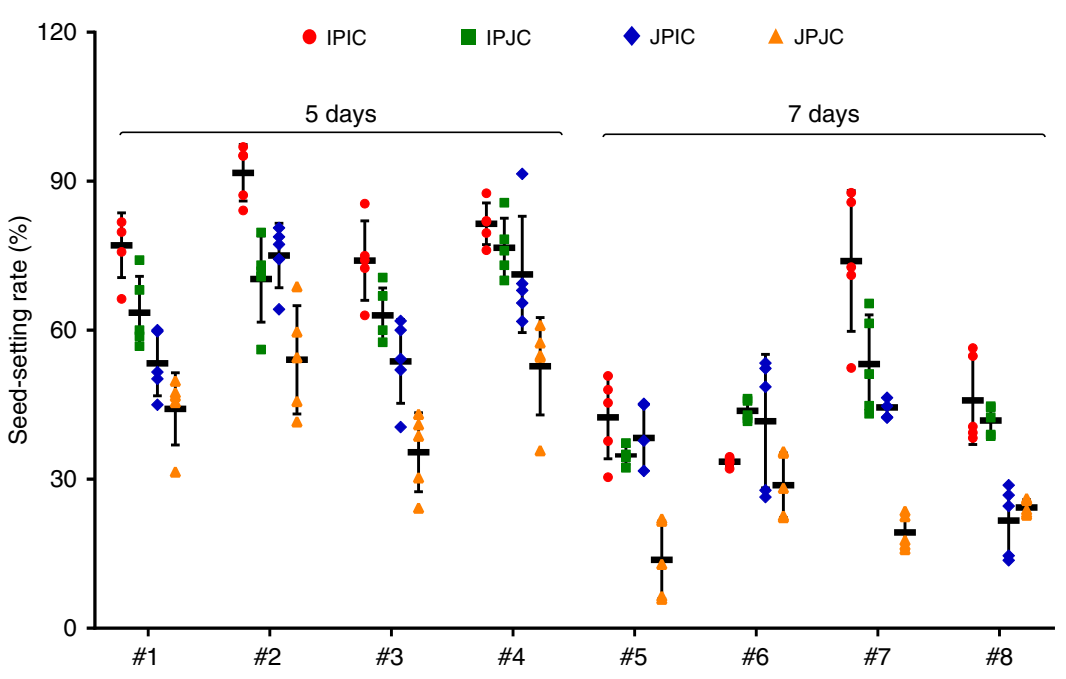

Fig. 6 SLGIInd enhances thermotolerance at the reproductive stage. a, $\mathbf{b}$ The slg1 mutant is highly sensitive to high temperature at the reproductive stage. Seed-setting rate was determined after treatment under $14 \mathrm{~h}$ light $\left(40^{\circ} \mathrm{C}\right) / 10 \mathrm{~h}$ dark $\left(31^{\circ} \mathrm{C}\right)$ for 5 days, and recovered under normal conditions until seed maturation. Data are means \pm SD $\left(n=10\right.$ panicles with three biological replicates). ${ }^{\star \star \star \star} P<0.0001$, two-tailed Student's $t$-test. Bar $=5 \mathrm{~cm}$. $\mathbf{c}$, $\mathbf{d}$ Comparison of thermotolerance among IPIC, IPJC, JPIC, and JPJC plants. Eight independent homozygous IPIC, IPJC, JPIC, and JPJC lines were treated with $14 \mathrm{~h}$ light $\left(40{ }^{\circ} \mathrm{C}\right) / 10 \mathrm{~h}$ dark $\left(31^{\circ} \mathrm{C}\right)$ for 5 days and 7 days, respectively, and then recovered under normal conditions until seed maturation. The $x$-axis indicates different transgenic lines. Data are means \pm SD ( $n=5$ panicles with three biological replicates). Bar $=5 \mathrm{~cm}$. The source data underlying (b), (d) are provided as a Source data file.

proteins, especially those involved in translation, metabolism, and stress responses (Supplementary Data 2 and Supplementary Fig. 19). The extensive influence of tRNA thiolation defects on protein homeostasis would trigger chronic proteotoxic stress ${ }^{27}$. Previous studies have shown that protein-synthesis defects in the URM1 pathway mutants in yeast led to the production of misfolded or error-containing proteins ${ }^{27,46}$, which triggered endoplasmic reticulum stress, thus activating downstream signaling pathways involved in abiotic-stress responses ${ }^{46,48}$. Similarly, our proteomic analysis also revealed the accumulation of five proteins (TrEMBL UniProt ID: Q6ZFJ9, Q651B0, Q653F6, Q6Z2M2, and Q9LWT6) in the slg1 mutant (Supplementary Data 2), these proteins appear to be involved in unfolded protein binding and chaperone-mediated protein folding. Moreover, we found that some oxidative stress-related proteins, such as TrEMBL UniProt ID Q0E4K1, Q0DTX5, P93407, and Q6L4W7 (Supplementary Data 2), were abnormally synthesized in the mutant, which might be due to the interplay between unfolded protein response and reactive oxygen species (ROS) signaling ${ }^{49}$. Therefore, the tRNA thiolation status in planta appears to be closely related to ROS homeostasis $^{38}$, which might be beneficial for the SLG1 $1^{\text {Ind }}$-carrying plants to tolerate high-temperature stress.

The two Asian cultivated subspecies, indica and japonica, are distributed worldwide from tropical to temperate regions. Typical indica and japonica cultivars have experienced local adaptation to different environmental niches during the long-term natural and artificial selection ${ }^{9}$. Recent works demonstrate that several genes such as COLD1, CTB4a, and bZIP73, contribute to the cold adaptation during japonica domestication ${ }^{10,11,13}$. Here, we showed that the SLG1 gene is differentiated between indica and japonica, and plants carrying SLG $1^{\text {Ind }}$ were more thermotolerant than those harboring $S L G 1^{T e j}$ at both seedling and reproductive stages, which was consistent with the distinct geographical distribution of the two subspecies. The sharply reduced nucleotide diversity of SLG1 strongly suggests that it may have been subjected to positive selection during domestication (Supplementary Fig. 9 and Supplementary Table 1). However, unlike other genes identified with specific functional nucleotide polymorphisms selected during domestication ${ }^{8,10,11,13}$, SLG1 showed variations in both promoter and coding sequences which collectively contribute to thermotolerance. Although the variations across SLG1 genomic region among wild rice accessions were highly abundant, most of the indica accessions carried Hap2 and were distributed predominantly in geographical regions with higher environmental temperature, suggesting that the domestication of indica rice was associated with strong selection of favored SLG1 alleles that enhanced thermotolerance for growth in regions with higher temperature (Fig. 7).

In summary, we demonstrate the role of tRNA thiolation in rice thermotolerance. Our results suggest that tRNA thiolation 


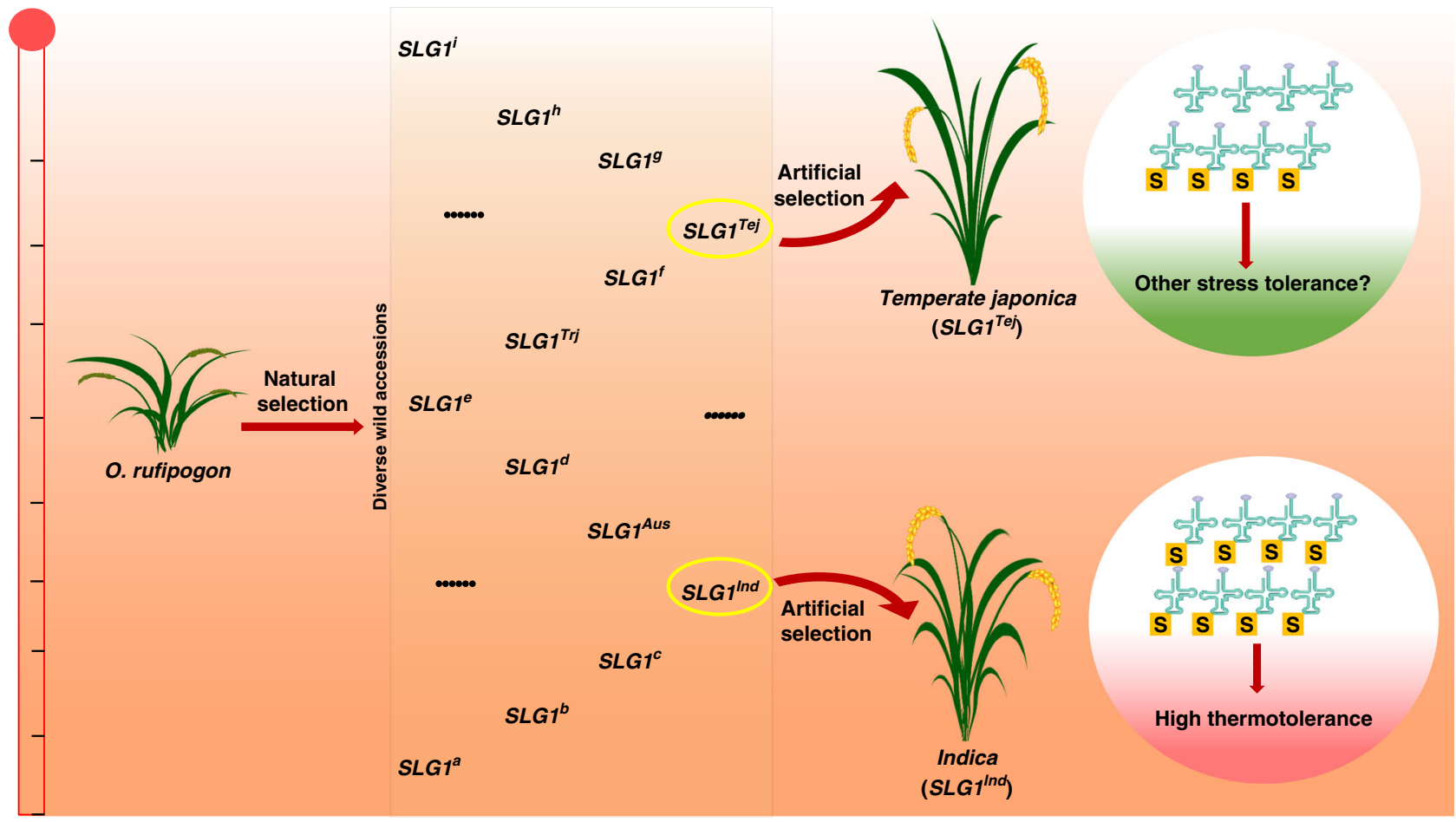

Fig. 7 Selection model of the SLG1 alleles during domestication. SLG1 is diversified among wild rice accessions. The intimate association of tRNA thiolation and thermotolerance led to the artificial selection of the SLGIInd allele for the adaptation of indica rice to geographical regions with high environmental temperature. Interestingly, the $S L G T^{T e j}$ allele was also subjected to selection during japonica domestication, which might be due to the dynamic response of tRNA thiolation to various environmental stresses ${ }^{46}$.

pathway might be a suitable target for breeding rice varieties with better tolerance to high-temperature stress. The greater diversity of SLG1 sequence in O. rufipogon wild rice accessions, combining with genome editing technique, may help us to select or generate excellent alleles with higher thermotolerance, which may contribute to the next generation rice breeding for the changing climate and warming globe.

\section{Methods}

Plant materials and growth conditions. The slg1 mutant was identified from the sodium azide mutagenized $\mathrm{M}_{2}$ population under the background of KY131, and the mapping population was derived from the cross of slg1 with KD8. For NIL construction, reciprocal cross was performed between ZF802 and KY131, and the resultant $F_{1}$ plants were backcrossed with ZF802 and KY131, respectively. The backcrossed progeny was genotyped with 111 polymorphic markers evenly distributed across the whole rice genome (Supplementary Fig. 20), and NIL-SLG1 ${ }^{T e j}$ and NIL-SLGI Ind were selected in the $\mathrm{BC}_{5} \mathrm{~F}_{2}$ generation. After two more rounds of self-fertilization, the homozygous $\mathrm{BC}_{5} \mathrm{~F}_{4}$ populations were used for phenotyping. For IPIC, IPJC, JPIC, and JPJC transgenic lines, 32 plants for each line were planted, and overall agronomic traits, including plant height, heading date, grain length, grain width, and seed-setting rate, were observed under normal growth conditions in three growing seasons, and the lines with WT-phenotype were selected for expression analysis and thermotolerance comparison. All the materials used in this study were cultivated in the experimental fields of the Institute of Genetics and Developmental Biology in Changping $\left(40.2^{\circ} \mathrm{N}, 116.2^{\circ} \mathrm{E}\right)$, Beijing during summer, and Lingshui $\left(18.5^{\circ} \mathrm{N}, 110.0^{\circ} \mathrm{E}\right)$, Hainan province during winter, respectively.

Thermotolerance assay. For high-temperature treatment at the seedling stage, healthy seeds were surface-sterilized with $3 \%$ sodium hypochlorite for $30 \mathrm{~min}$, soaked at $37^{\circ} \mathrm{C}$ for 3 days. Germinating seeds were sowed into 96-well plates, and water-cultured in the phytotron (SANYO) with $14 \mathrm{~h}$ light $\left(28^{\circ} \mathrm{C}\right) / 10 \mathrm{~h}$ dark $\left(25^{\circ} \mathrm{C}\right), 65-70 \%$ relative humidity, and $150 \mu \mathrm{M} \mathrm{m}^{-2} \mathrm{~s}^{-1}$ photon flux density. Fiveday-old pre-cultured seedlings were then transferred to outdoor natural conditions for further growth. Seedlings of 2-leaf stage were treated with $45^{\circ} \mathrm{C} \pm 1{ }^{\circ} \mathrm{C}$ for a given time in the above-described phytotron (SANYO), and then recovered under normal conditions for 10 days. The survival rate was calculated as the ratio of the number of seedlings with new green leaves to the total number of treated seedlings.
For high-temperature treatment at the reproductive stage, germinating seeds were sowed on April 16, April 26, and May 12, respectively, and the seedlings of 4leaf stage were transplanted into pots $(25 \mathrm{~cm} \times 25 \mathrm{~cm} \times 35 \mathrm{~cm})$ filled with the same amount of paddy soil, each pot with four plants, and cultivated under natural growth conditions. The tillers at the microsporocyte meiosis stage were tagged and transferred into the phytotron (PERCIVAL, USA), with the photon flux density of $500 \mu \mathrm{mol} \mathrm{m}-2 \mathrm{~s}^{-1}$ and relative humidity of $75-80 \%$. High-temperature treatment was performed under $40^{\circ} \mathrm{C}$ during the day (06:00-20:00) and $31{ }^{\circ} \mathrm{C}$ at night (20:00-06:00) for 5 and 7 days, respectively, and the plants were then recovered under normal growth conditions until seed maturation. Seed-setting rate was calculated as the percentage of the number of filled grains to the number of total spikelets in a panicle.

Map-base cloning. The mapping population was derived from the cross of $\lg 1$ with KD8, and whole-genome polymorphic markers were designed based on resequencing data $(30 \times)$. Using 20 bulked WT and mutant plants, respectively, the candidate gene was first mapped to the long arm of chromosome 12 between markers $\mathrm{C} 1$ and $\mathrm{C} 5$. Further analysis of the $\mathrm{F}_{2}$ mutant plants subsequently finemapped the casual gene to the region between $\mathrm{C} 4$ and C6. Sequence comparisons between slg1 and WT were then performed for all the annotated genes within this region, and a single-nucleotide substitution ( $\mathrm{G}$ to A) at the splicing acceptor site of the fifth intron of LOC_Os12g39840 was identified in the mutant. This mutation led to the formation of at least four kinds of splicing variants of SLG1 in the mutant which were confirmed by sequencing, and no normal SLG1 transcript was found in the mutant. The primer set used for amplifying the splicing variants is listed in Supplementary Data 3.

Plasmid construction and plant transformation. For JPJC and IPIC complementary plasmids, a SLG1 genomic DNA containing the $1637 \mathrm{bp}$ fragment upstream of ATG, coding region, and 840 bp downstream sequence was amplified from KY131 (SLG1 $\left.{ }^{T e j}\right)$ and 93-11 (SLGI $\left.{ }^{I n d}\right)$, respectively, and then ligated into the binary vector pZH2B using XbaI and SacI. For JPIC and IPJC constructs, $\mathrm{XbaI}$ and $A p a I$ were first used to cut the JPJC and IPIC complementary plasmids, respectively, to obtain the promoter sequence of $S L G 1^{T e j}$ and $S L G 1^{I n d}$ alleles. Next, ApaI and SacI were used to cut the JPJC and IPIC plasmids, respectively, to obtain the rest of the DNA fragment. Then, the SLGI Tej promoter and the rest of SLGI Ind DNA fragment were integrated with $\mathrm{T}_{4}$ ligase to produce the JPIC construct, and the SLGI Ind promoter and the rest of SLG1 $1^{T e j}$ DNA fragment were ligated to generate IPJC construct (Supplementary Fig. 13a). For SLG1-RNAi and RCTU1RNAi plasmids, the hairpin sequence with two $\sim 300$ bp cDNA inverted repeats was inserted into the binary vector $\mathrm{pZH} 2 \mathrm{Bi}$, and introduced into slg1 and $\mathrm{KY} 131$ 
background, respectively. For overexpression construct, the full-length coding sequence of SLG1 was amplified from KY131 and ligated into pZH2Bi driven by the ubiquitin promoter, and introduced into the KY131 background. For Flagtagged SLG1 overexpressing plasmid, the full-length coding sequence of $S L G 1$ was amplified from KY131 and ligated into Cambia 1300-221-Flag vector driven by the $35 \mathrm{~S}$ promoter, then introduced into the slg1 mutant background. Primers used for vector construction are listed in Supplementary Data 3. Rice transformation was carried out by Agrobacterium-mediated method.

Quantitative real-time RT-PCR. Total RNA was isolated from different tissues using the RNAiso Plus Kit (TaKaRa). About $1 \mu \mathrm{g}$ RNA was treated with DNase I (Promega) and reversely transcribed using a reverse transcription kit (Promega). The qRT-PCR assay was carried out using SYBR Green I Master reagent and a Light Cycler Nano system (Roche). The Actin gene was used as the internal control for normalization. Primers used for expression analysis are listed in Supplementary Data 3.

Microscopic examination. For root hair examination, healthy seeds were sterilized by $2 \%$ sodium hypochlorite for $30 \mathrm{~min}$, and then sowed in $1 / 2 \mathrm{MS}$ solid medium for 3 days under the photoperiod of $14 \mathrm{~h}$ light $\left(28^{\circ} \mathrm{C}\right) / 10 \mathrm{~h}$ dark $\left(25^{\circ} \mathrm{C}\right)$. The seminal roots of about $1 \mathrm{~cm}$ in length were cut off and immersed in the liquid nitrogen, and root hair morphology was observed under a cryo-scanning electron microscope (S-3000N, Hitachi).

Anthers and florets were collected from the plants grown under normal and high-temperature $\left(40^{\circ} \mathrm{C}, 5\right.$ days) conditions, respectively, at the flowering stage. The anthers were then stained with $1 \% \mathrm{I}_{2}$-KI solution to evaluate pollen fertility, and the florets were stained with $0.5 \%$ aniline blue solution for $12 \mathrm{~h}$ to observe the amount of pollens on the stigmas. To visualize pollen-tube growth, the pistils from the plants $\sim 30 \mathrm{~min}$ after flowering grown under normal and high-temperature conditions were collected, and fixed in the solution containing 30\% chloroform, $10 \%$ acetic acid, and $57 \%$ ethanol. After washing with deionized water, pistils were incubated in $10 \mathrm{~mol} / \mathrm{L} \mathrm{NaOH}$ under $56^{\circ} \mathrm{C}$ for $8 \mathrm{~min}$ and stained with $0.1 \%$ aniline blue solution for $12 \mathrm{~h}$. The above samples were then photographed under the wavelength of $350 \mathrm{~nm}$ using a microscope (BX53, Olympus)

tRNA extraction and quantification. Shoots of 50 seedlings at 2-leaf-stage grown under normal and high-temperature $\left(45^{\circ} \mathrm{C}, 44 \mathrm{~h}\right)$ conditions, respectively, were collected for each extraction. tRNA was extracted according to the previously described method with some modifications ${ }^{36}$. Briefly, samples were ground in liquid nitrogen and suspended in $6 \mathrm{~mL} 0.9 \% \mathrm{NaCl}$, and the suspension was transferred to an RNAase-free 50-mL falcon tube. After adding $12 \mathrm{~mL}$ acid phenol (Sigma) and $1.2 \mathrm{~mL}$ chloroform, and mixing in a vortex for $30 \mathrm{~s}$, the mixture was centrifuged at $8600 \times g$ for $10 \mathrm{~min}$. Then, the supernatant was transferred to a new $50-\mathrm{mL}$ falcon tube with the addition of $6 \mathrm{~mL}$ acidic phenol and $0.6 \mathrm{~mL}$ chloroform. Repeat the above steps for 2-3 times until the interphase was moderately clean. After equilibrating with $10 \mathrm{~mL}$ buffer [ $10 \mathrm{mM}$ Tris ( $\mathrm{pH}$ 6.3), $15 \%$ ethanol, $200 \mathrm{mM}$ $\mathrm{KCl}, 0.15 \%$ Triton X-100], the Nucleobond AX100 columns (Macherey Nagel) were then used to bind the tRNA in the above supernatant. After washing twice with $12 \mathrm{~mL}$ buffer [ $10 \mathrm{mM}$ Tris ( $\mathrm{pH} 6.3$ ), $15 \%$ ethanol, $300 \mathrm{mM} \mathrm{KCl}$, the column were then eluted with $10 \mathrm{~mL}$ elution buffer $[10 \mathrm{mM}$ Tris (pH 6.3), 15\% ethanol, $650 \mathrm{mM} \mathrm{KCl}$, and the effluent was collected into a new falcon tube containing $25 \mathrm{~mL}$ absolute ethanol and gently mixed. After standing overnight at $-20^{\circ} \mathrm{C}$, the mixture was centrifuged at $8600 \times g$ for $10 \mathrm{~min}$ at room temperature. The precipitate was then washed 2-3 times with $80 \%$ ethanol, dried at room temperature, and dissolved with $40 \mu \mathrm{L}$ DEPC $\mathrm{H}_{2} \mathrm{O}$

For APM-PAGE, the above extracted tRNA was denatured at $80^{\circ} \mathrm{C}$ for $5 \mathrm{~min}$ and separated on a $10 \%$ acrylamide gel supplemented with $50 \mu \mathrm{g} / \mathrm{mL}$ APM (TRC, Canada). The resulting gel was stained with ethidium bromide and photographed using the Bio Doc-It ${ }^{\mathrm{TM}}$ imaging system.

For Northern blots, tRNAs from WT and slg1 were separated by APM-PAGE gels as described above, and the gel was transferred to positively charged Nylon membrane (Roche) with Semi-dry Transfer System (BIO-RAD, 170-3940). Then the membrane was blotted following the instructions for DIG Northern Starter Kit (Roche). The DNA probes were synthesized following the sequences listed in Supplementary Data 3 and DIG-labeled (Roche).

For LC/MS-QQQ analysis, about $40 \mu \mathrm{g}$ tRNA from different samples was digested with Nuclease P1 (Sigma: N8630) and bacterial alkaline phosphatase (Sigma: P4252), and the hydrolysate diluted with an equal volume of water was then analyzed by LC/MS-QQQ. Calibration curve of $\mathrm{mcm}^{5} \mathrm{~s}^{2} \mathrm{U}$ was obtained with different concentrations of authentic standard. The amount of $\mathrm{mcm}^{5} \mathrm{~s}^{2} \mathrm{U}$ in standard samples was determined by the linear regression of the peak area vs its concentration. And the $\mathrm{mcm}^{5} \mathrm{~s}^{2} \mathrm{U}$ concentration in individual samples was quantified with the calibration curve. Chromatographic separation was performed on a ZORBAX Elipse Plus C18 RRHD column $(100 \mathrm{~mm} \times 2.1 \mathrm{~mm}$ ID, $1.8 \mu \mathrm{m}$, Agilent). The amount of tRNA sample loaded onto the column was $1 \mu \mathrm{L}(0.286 \mu \mathrm{g} /$ $\mu \mathrm{L})$. Mobile phase was as following: solution A ( $10 \%$ acetonitrile, $5 \mathrm{mM}$ ammonium formate); solution B (90\% acetonitrile, $5 \mathrm{mM}$ ammonium formate). Flow rate was $0.35 \mathrm{~mL} / \mathrm{min}$. Gradient condition of the mobile phase was as follows: 0-0.5 min, $100 \%$ solution A; $0.5-3.0$ min, $100 \%$ B; $3.0-4.0$ min, $100 \%$ buffer B. Data were acquired by 1290 UPLC/G6495A QQQ MS (Agilent, USA) in MRM mode.
The transitions from precursor ions to product ion are $\mathrm{m} / z 333$ to $201(\mathrm{CE}=10 \mathrm{v})$ in positive mode for $\mathrm{mcm}^{5} \mathrm{~s}^{2} \mathrm{U}$. The parameters of MS detection were as following: source temperature is $100^{\circ} \mathrm{C}$; gas temperature is $200^{\circ} \mathrm{C}$; gas flow is $15 \mathrm{~L} / \mathrm{min}$; nebulizer is $30 \mathrm{psi}$; sheath gas heater temperature is $350{ }^{\circ} \mathrm{C}$; sheath gas flow is $12 \mathrm{~L} /$ min. The data are processed by the Masshunter software.

Transient expression in rice protoplasts. For subcellular localization, the fulllength coding sequences of SLG1 and AtCTU2 were cloned into PSAT6-EYFP-N1 vector to form SLG1-EYFP and AtCTU2-EYFP constructs, respectively, and empty vector was used as a negative control. OsbZIP52-RFP was used as the nuclear localization marker. For BiFC assay, the full-length coding sequences of SLG1 and RCTU1 were cloned into pUC19-VYNE(R) and $\mathrm{pUC19-VYCE}(\mathrm{R})$ vectors and fused with either $\mathrm{N}$ - or C-terminal of Venus sequence, respectively. These constructs and corresponding empty vectors were transformed into rice protoplasts. After incubation in dark for $16 \mathrm{~h}$, YFP, RFP, and Venus signals were observed using laser scanning confocal microscope (Leica TCS SP5).

For promoter activity assay, the SLG1 promoter sequences used for the complementary test were amplified from Nipponbare ( $\left.p S L G 1^{T e j}\right)$ and $93-11$ $\left(p S L G 1^{I n d}\right)$, respectively, and cloned into pGreenII 0800-LUC vector containing the firefly luciferase (fLUC) gene and the Renilla LUC gene (rLUC). These constructs and corresponding empty vectors were transformed into rice protoplasts, respectively. The promoter of Chalk 5 was used as a positive control ${ }^{50}$. After incubation in dark for $16 \mathrm{~h}$ protoplasts were collected and luciferase activity was measured as described in the Dual-Luciferase ${ }^{\circledast}$ Reporter Assay System Manual (Promega).

Yeast two-hybrid assay. The full-length coding sequences of SLG1 and RCTU1 were amplified from KY131 and cloned into pGBKT7 or pGADT7 vector (Clontech), respectively. The resulting constructs and the corresponding empty vectors were transformed into Golden Yeast according to the Clontech Yeast Two-Hybrid System User Manual, and interactions were detected on SD/-Leu-Trp-His-Ade medium.

Co-immunoprecipitation. Rice protoplasts from Flag-tagged transgenic plants overexpressing SLG1 were transformed with plasmid containing $6 \times$ Myc-tagged RCTU1 expression cassette. After $16 \mathrm{~h}$ incubation, the protoplasts were collected by centrifugation at $150 \times g$ and the pellet was resuspended with protein extraction buffer [50 mM Tris- $\mathrm{HCl}$ (pH 7.5), $150 \mathrm{mM} \mathrm{NaCl}, 5 \mathrm{mM}$ EDTA, 0.1\% Triton X-100, $0.2 \%$ Nonidet P 40, 0.6 mM PMSF, and $1 \times$ Protein Inhibitor Cocktail (Roche)]. After 30 min gentle rotation at $4{ }^{\circ} \mathrm{C}$, the lysate was centrifuged at $4{ }^{\circ} \mathrm{C}$ with $14,000 \times g$, and the supernatant was co-incubated for $16 \mathrm{~h}$ with anti-Flag beads (Merck, M8823-1ML) prewashed with protein extraction buffer. The beads were then washed five times with protein extraction buffer and boiled in $1 \times$ SDS sample buffer for $5 \mathrm{~min}$. The supernatant was used for western blotting analysis. Anti-flag (Sigma, F1804) and anti-Myc (Abcam, ab9106) were used for the detection of immunoprecipitated flag-tagged and Myc-tagged protein in the western blot analysis, respectively. The antibodies were all diluted by 1000 times.

Haplotype analysis. The 1637-bp promoter and full-length coding sequence of SLG1 in 4219 cultivated rice accessions were retrieved from the rice VarMap2 database (http://ricevarmap.ncpgr.cn/v2/4 ${ }^{40}$, and haplotype analysis was carried out using the method in the database. Only haplotypes found in $\geq 10$ rice accessions were recorded. The wild rice sequence information was collected from the Rice Annotation Project database (https://ricegenome.dna.affrc.go.jp/). Haplotype information of Mini-Core germplasms collected worldwide by US Department of Agriculture ${ }^{51}$ were used to plot on the map, in which the 30-year average terrestrial temperature obtained from Esri (https://www.esri.com) were also plotted using ArcGIS (http://www.arcgis.com/)

Nucleotide diversity and fixation index calculation. Nucleotide diversity of $S L G 1$ and surrounding regions were calculated on the Mini-Core dataset using a custom Perl script (https://github.com/oushujun/ThetaPi) with parameters '-missing_rate 1 -window_size $100^{\prime 52}$. Fixation index $\left(F_{\mathrm{ST}}\right)$ was calculated using VCFtools with 1000 -bp windows and 100-bp steps on the 20-kb region and 10-kp windows and 1$\mathrm{kb}$ steps on the $2-\mathrm{Mb}$ region $^{53,54}$

Phylogenetic analysis. The entire amino-acid sequence of SLG1 was used as the query to search for the homologous proteins in the NCBI website (https://blast. ncbi.nlm.nih.gov/Blast.cgi), and phylogenetic analysis was performed using MEGA7.0. The neighbor-joining method was used with 1000 bootstrap replications. For phylogenetic reconstruction of $S L G 1$, the neighbor-joining trees were constructed using MEGA7.0 with pair-wise deletion for missing data.

Proteomics and bioinformatics analysis. Shoots of 40 seedlings of slg1 and WT at 2-leaf stage grown under normal conditions were sampled for protein extraction. The differentially expressed proteins were identified by using iTRAQ-based quantitative proteomics and LC-MS/MS by Shanghai OE Biotechnology Company (http://www.shoysw.cn/). In summary, 1610 proteins were quantified (FDR $<1 \%$ ), and 112 differentially expressed proteins were selected for $>1.2$-fold change 
between slg1 and WT. The annotation of the differentially expressed proteins between slg1 and WT was based on the GO annotations of molecular function (http://www.geneontology.org/).

Comparison of thiolase domain was performed between SLG1 and the known thiolase domain containing proteins of AtCTU2 from Arabidopsis thaliana (UniProt: O65628), ScCTU2 from Saccharomyces cerevisiae (UniProt: P53923), and HsCTU2 from Homo sapiens (UniProt: Q2VPK5). Sequence alignment was conducted on the website of ClustalW (https://www.genome.jp/tools-bin/clustalw).

\section{Data availability}

Data supporting the findings of this work are available within the paper and its Supplementary Information files. A reporting summary for this Article is available as a Supplementary Information file. The datasets and plant materials generated and analyzed during the current study are available from the corresponding author upon request. The mass spectrometry proteomics data have been deposited to the iProX repositories with the dataset identifier IPX0002504000. Source data are provided with this paper.

Received: 28 February 2020; Accepted: 6 October 2020; Published online: 28 October 2020

\section{References}

1. Zhao, C. et al. Plausible rice yield losses under future climate warming. Nat. Plants 3, 16202 (2016).

2. Sreenivasulu, N. et al. Designing climate-resilient rice with ideal grain quality suited for high-temperature stress. J. Exp. Bot. 66, 1737-1748 (2015).

3. De Storme, N. \& Geelen, D. The impact of environmental stress on male reproductive development in plants: biological processes and molecular mechanisms. Plant Cell Environ. 37, 1-18 (2014).

4. Fu, G. et al. Heat stress is more damaging to superior spikelets than inferiors of rice (Oryza sativa L.) due to their different organ temperatures. Front. Plant Sci. 7, 1637 (2016).

5. Zhang, C. et al. Heat stress induces spikelet sterility in rice at anthesis through inhibition of pollen tube elongation interfering with auxin homeostasis in pollinated pistils. Rice 11, 14 (2018).

6. Nevame, A. Y. M. et al. Relationship between high temperature and formation of chalkiness and their effects on quality of rice. Biomed. Res. Int. 2018, 1653721 (2018)

7. Zhang, H., Xu, H., Feng, M. \& Zhu, Y. Suppression of OsMADS7 in rice endosperm stabilizes amylose content under high temperature stress. Plant Biotechnol. J. 16, 18-26 (2018).

8. $\mathrm{Li}, \mathrm{X}$. M. et al. Natural alleles of a proteasome $\alpha 2$ subunit gene contribute to thermotolerance and adaptation of African rice. Nat. Genet. 47, 827-833 (2015).

9. Gardener, C. \& Kumar, S. V. Hot n' cold: molecular signatures of domestication bring fresh insights into environmental adaptation. Mol. Plant. 8, 1439-1441 (2015).

10. Liu, C. et al. Early selection of bZIP73 facilitated adaptation of japonica rice to cold climates. Nat. Commun. 9, 3302 (2018)

11. Ma, Y. et al. COLD1 confers chilling tolerance in rice. Cell 160, 1209-1221 (2015).

12. Liu, C. et al. The $b Z I P 73$ transcription factor controls rice cold tolerance at the reproductive stage. Plant Biotechnol. J. 17, 1834-1849 (2019).

13. Zhang, Z. et al. Natural variation in CTB4a enhances rice adaptation to cold habitats. Nat. Commun. 8, 14788 (2017).

14. Chen, K. et al. Translational regulation of plant response to high temperature by a dual-function $\mathrm{tRNA}^{\mathrm{His}}$ guanylyltransferase in rice. Mol. Plant. 12, 1123-1142 (2019).

15. Gupta, R. et al. A tRNA modification balances carbon and nitrogen metabolism by regulating phosphate homeostasis. Elife 8, e44795 (2019).

16. Wang, Y. et al. Identification of tRNA nucleoside modification genes critical for stress response and development in rice and Arabidopsis. BMC Plant Biol. 17, 261 (2017).

17. Yacoubi, B. E., Bailly, M. \& Crécy-Lagard, V. D. Biosynthesis and function of posttranscriptional modifications of transfer RNAs. Annu. Rev. Genet. 46, 69-95 (2012)

18. Bjork, G. R., Huang, B., Persson, O. P. \& Bystrom, A. S. A conserved modified wobble nucleoside $\left(\mathrm{mcm}^{5} \mathrm{~s}^{2} \mathrm{U}\right)$ in lysyl-tRNA is required for viability in yeast. RNA 13, 1245-1255 (2007).

19. Karlsborn, T. et al. Elongator, a conserved complex required for wobble uridine modifications in eukaryotes. RNA Biol. 11, 1519-1528 (2014).

20. Leidel, S. et al. Ubiquitin-related modifier Urml acts as a sulphur carrier in thiolation of eukaryotic transfer RNA. Nature 458, 228-232 (2009).
21. Rezgui, V. A. et al. $t R N A t \mathrm{~K}^{\mathrm{UUU}}, \mathrm{tQ}^{\mathrm{UUG}}$, and $\mathrm{tE}^{\mathrm{UUC}}$ wobble position modifications fine-tune protein translation by promoting ribosome A-site binding. Proc. Natl Acad. Sci. USA 110, 12289-12294 (2013).

22. Termathe, M. \& Leidel, S. A. The Uba4 domain interplay is mediated via a thioester that is critical for tRNA thiolation through Urm1 thiocarboxylation. Nucleic Acids Res. 46, 5171-5181 (2018).

23. Judes, A., Bruch, A., Klassen, R., Helm, M. \& Schaffrath, R. Sulfur transfer and activation by ubiquitin-like modifier system Uba4.Urm1 link protein urmylation and tRNA thiolation in yeast. Microb. Cell 3, 554-564 (2016).

24. Dewez, M. et al. The conserved wobble uridine tRNA thiolase Ctu1-Ctu2 is required to maintain genome integrity. Proc. Natl Acad. Sci. USA 105, 5459-5464 (2008)

25. Cavuzic, M. \& Liu, Y. Biosynthesis of sulfur-containing tRNA modifications: a comparison of bacterial, archaeal, and eukaryotic pathways. Biomolecules 7, 27 (2017).

26. Shaheen, R. et al. Biallelic variants in CTU2 cause DREAM-PL syndrome and impair thiolation of tRNA wobble U34. Hum. Mutat. 40, 2108-2120 (2019).

27. Nedialkova, D. D. \& Leidel, S. A. Optimization of codon translation rates via tRNA modifications maintains proteome integrity. Cell 161, 1606-1618 (2015).

28. Laxman, S. et al. Sulfur amino acids regulate translational capacity and metabolic homeostasis through modulation of tRNA thiolation. Cell 154, 416-429 (2013).

29. Goehring, A. S., Rivers, D. M. \& Sprague, G. F. Jr. Urmylation: a ubiquitin-like pathway that functions during invasive growth and budding in yeast. Mol. Biol. Cell 14, 4329-4341 (2003).

30. Fernandez-Vazquez, J. et al. Modification of tRNA ${ }^{\text {Lys }}$ UuU by elongator is essential for efficient translation of stress mRNAs. PLoS Genet. 9, e1003647 (2013).

31. Zinshteyn, B. \& Gilbert, W. V. Loss of a conserved tRNA anticodon modification perturbs cellular signaling. PLoS Genet. 9, e1003675 (2013).

32. Chavarria, N. E. et al. Archaeal Tuc1/Ncs6 homolog required for wobble uridine tRNA thiolation is associated with ubiquitin-proteasome, translation, and RNA processing system homologs. PLoS ONE 9, e99104 (2014).

33. Alings, F., Sarin, L. P., Fufezan, C., Drexler, H. C. \& Leidel, S. A. An evolutionary approach uncovers a diverse response of tRNA 2-thiolation to elevated temperatures in yeast. RNA 21, 202-212 (2015).

34. Sinha, H. et al. Sequential elimination of major-effect contributors identifies additional quantitative trait loci conditioning high-temperature growth in yeast. Genetics 180, 1661-1670 (2008)

35. Tyagi, K. \& Pedrioli, P. G. Protein degradation and dynamic tRNA thiolation fine-tune translation at elevated temperatures. Nucleic Acids Res. 43, 4701-4712 (2015)

36. Philipp, M., John, F. \& Ringli, C. The cytosolic thiouridylase CTU2 of Arabidopsis thaliana is essential for posttranscriptional thiolation of tRNAs and influences root development. BMC Plant Biol. 14, 109 (2014).

37. Nakai, Y., Nakai, M. \& Yano, T. Sulfur modifications of the wobble $U_{34}$ in tRNAs and their intracellular localization in Eukaryotic cells. Biomolecules 7, 17 (2017).

38. Leiber, R. M. et al. The TOR pathway modulates the structure of cell walls in Arabidopsis. Plant Cell 22, 1898-1908 (2010).

39. Kovach, M. J., Sweeney, M. T. \& McCouch, S. R. New insights into the history of rice domestication. Trends Genet 23, 578-587 (2007).

40. Zhao, H. et al. RiceVarMap: a comprehensive database of rice genomic variations. Nucleic Acids Res. 43, D1018-D1022 (2015).

41. Huang, X. et al. A map of rice genome variation reveals the origin of cultivated rice. Nature 490, 497-501 (2012).

42. Agrama, H. A. et al. Genetic assessment of a Mini-Core subset developed from the USDA rice genebank. Crop Sci. 49, 1336-1346 (2009).

43. Reig-Valiente, J. L. et al. Genetic diversity and population structure of rice varieties cultivated in temperate regions. Rice 9, 58 (2016).

44. Endo, M. et al. High temperatures cause male sterility in rice plants with transcriptional alterations during pollen development. Plant Cell Physiol. 50, 1911-1922 (2009)

45. Kubo, T., Takashi, T., Ashikari, M., Yoshimura, A. \& Kurata, N. Two tightly linked genes at the $h s a 1$ locus cause both $\mathrm{F}_{1}$ and $\mathrm{F}_{2}$ hybrid sterility in rice. Mol. Plant. 9, 221-232 (2016).

46. Damon, J. R., Pincus, D. \& Ploegh, H. L. tRNA thiolation links translation to stress responses in Saccharomyces cerevisiae. Mol. Biol. Cell 26, 270-282 (2015).

47. Bruch, A., Klassen, R. \& Schaffrath, R. Unfolded protein response suppression in yeast by loss of tRNA modifications. Genes (Basel) 9, 516 (2018).

48. Afrin, T., Diwan, D., Sahawneh, K. \& Pajerowska-Mukhtar, K. Multilevel regulation of endoplasmic reticulum stress responses in plants: where old roads and new paths meet. J. Exp. Bot. 71, 1659-1667 (2019).

49. Ozgur, R., Uzilday, B., Iwata, Y., Koizumi, N. \& Turkan, I. Interplay between the unfolded protein response and reactive oxygen species: a dynamic duo. $J$. Exp. Bot. 69, 3333-3345 (2018). 
50. Li, Y. et al. Chalk5 encodes a vacuolar $\mathrm{H}^{+}$-translocating pyrophosphatase influencing grain chalkiness in rice. Nat. Genet. 46, 398-404 (2014).

51. Wang, H. et al. The power of inbreeding: NGS-based GWAS of rice reveals convergent evolution during rice Domestication. Mol. Plant. 9, 975-985 (2016).

52. Wang, H., Vieira, F. G., Crawford, J. E., Chu, C. \& Nielsen, R. Asian wild rice is a hybrid swarm with extensive gene flow and feralization from domesticated rice. Genome Res. 27, 1029-1038 (2017).

53. Weir, B. S. \& Cockerham, C. C. Estimating F-statistics for the analysis of population structure. Evolution 38, 1358-1370 (1984).

54. Danecek, P. et al. The variant call format and VCFtools. Bioinformatics 27, 2156-2158 (2011).

\section{Acknowledgements}

The plant tRNA extraction method was kindly provided by Professor Christoph Ringli (Institute of Plant Biology, University of Zürich). The $\mathrm{mcm}^{5} \mathrm{~s}^{2} \mathrm{U}$ standard was a generous gift by Professor Anders S. Byström (Department of Molecular Biology, Umeå University). We thank Dr. Fu Xu (University of Umeå), Professor Sebastian A. Leidel (Max Planck Institute for Molecular Biomedicine), and Dr. Sabrina M. Huber (Massachusetts Institute of Technology) for their advices in tRNA purification and quantification. We are also grateful to Dr. Fengxia Zhang (Professor Guodong Wang's lab, Institute of Genetics and Developmental Biology, CAS) for the help in quantification of thiolated tRNAs. This work was supported by the grants from the National Key Research and Development Program of China (Grant: 2016YFD0101801), the Strategic Priority Research Program of the Chinese Academy of Sciences (XDA24030201), and the State Key Laboratory of Plant Genomics.

\section{Author contributions}

S.Y. supervised the project; S.Y., Y.X., and C.C. designed the research; Y.X. and L.Z. performed most of the experiment; S.O. analyzed the nucleotide diversity, fixation index, phylogenetic reconstruction and haplotype distribution; R.W. isolated the mutant and performed field management; Y.W. contributed the reagents and experimental support; Y.X., S.Y., and C.C. analyzed the data; Y.X., S.Y., C.C., and S.O. wrote the paper.

\section{Competing interests}

The authors declare no competing interests.

\section{Additional information}

Supplementary information is available for this paper at https://doi.org/10.1038/s41467020-19320-9.

Correspondence and requests for materials should be addressed to C.C. or S.Y.

Peer review information Nature Communications thanks the anonymous reviewers for their contribution to the peer review of this work. Peer reviewer reports are available.

Reprints and permission information is available at http://www.nature.com/reprints

Publisher's note Springer Nature remains neutral with regard to jurisdictional claims in published maps and institutional affiliations.

cc (i) Open Access This article is licensed under a Creative Common Attribution 4.0 International License, which permits use, sharing, adaptation, distribution and reproduction in any medium or format, as long as you give appropriate credit to the original author(s) and the source, provide a link to the Creative Commons license, and indicate if changes were made. The images or other third party material in this article are included in the article's Creative Commons license, unless indicated otherwise in a credit line to the material. If material is not included in the article's Creative Commons license and your intended use is not permitted by statutory regulation or exceeds the permitted use, you will need to obtain permission directly from the copyright holder. To view a copy of this license, visit http://creativecommons.org/ licenses/by/4.0/.

(c) The Author(s) 2020 